\title{
Recent developments in guanylating agents
}

\author{
Alan R. Katritzky**, and Boris V. Rogovoy ${ }^{\S}$ \\ ${ }^{ \pm}$Center for Heterocyclic Compounds, Department of Chemistry, University of Florida, P. O. \\ Box 177200, Gainesville, FL 32611-7200 \\ $\S^{\S}$ Department of Pharmaceutical Chemistry, Ernest Mario School of Pharmacy, Rutgers, The \\ State University of New Jersey, 160 Frelinghuysen Road, Piscataway, NJ 08854 \\ E-mail: katritzky@chem.ufl.edu
}

\section{Dedicated to Professor Nikolai Zefirov on the occasion of his $70^{\text {th }}$ birthday (received 26 Oct 04; accepted 08 Jan 05; published on the web 05 Jan 05)}

\begin{abstract}
Guanidines are important molecules with a wide range of interesting properties. In this overview we summarize recent advances in the development of guanylating reagents which we define as compounds forming a guanidine structure by a chemical transformation. We cover important classes of guanylating agents developed in the last two decades and representative examples are reported.
\end{abstract}

Keywords: Guanidine, guanidines, guanylating agent, guanylation, synthesis, preparation

\section{Contents}

1. Introduction

2. Reagents for the Preparation of Guanidines

2.1. Thioureas

2.2. Isothioureas

2.3. Carbodiimides and Cyanamides

2.4. Pyrazole-1-carboximidamides

2.5. Triflyl guanidines

2.6. Aminoiminomethane -sulfonic and -sulfinic acids

2.7. Benzotriazole and Imidazole-containing Reagents

3. Conclusions

4. References

\section{Introduction}

Guanidines possess great biochemical and pharmaceutical importance. Guanidine itself is a strong base (pKa of conjugated acid is 12.5) as are substituted guanidines. ${ }^{\text {1a }}$ Guanidine was first prepared by Strecker in 1861 by oxidizing guanine. The biological role, chemical and biochemical properties of natural and synthetic guanidine derivatives have been outlined. ${ }^{1 \mathrm{~b}}$ The 
natural amino acid, L-arginine 1, is often found at active (or catalytic) sites in proteins and enzymes; it is critical for the normal function of living organisms. ${ }^{2}$

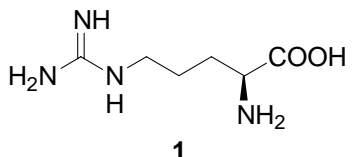

Many natural guanidines have been isolated and tested for biological activity. The isolation, structural identification and synthesis of naturally occurring guanidines have been reviewed by Berlinck. $^{3 a-d}$ The isolation and synthesis of guanidine metabolites from Ptilocaulis spiculifer has also been summarized. ${ }^{3 e}$ Isolation of guanidine compounds as metabolites provides leads for the prevention of metabolic disorders and helps the prognosis of cancer, cardiovascular diseases, diabetes etc. ${ }^{3 \mathrm{f}}$ Recent examples of synthetic, biologically-active guanidines include antimicrobial activity, ${ }^{4 a, b}$ thrombin inhibitors, ${ }^{5} \mathrm{Na}^{+} / \mathrm{H}^{+}$exchanger (NHE) inhibitors, ${ }^{6 \mathrm{a}, \mathrm{b}}$ transport for the delivery of anti-cancer agents, ${ }^{7 \mathrm{a}, \mathrm{b}}$ anti-influenza agents. ${ }^{7 \mathrm{~d}}$

Figure 1 shows a few examples of superpotent sweeteners with the guanidine core structure; ${ }^{8 \mathrm{a}}$ which have attracted a great deal of recent interest. ${ }^{8 \mathrm{~b}-\mathrm{e}}$

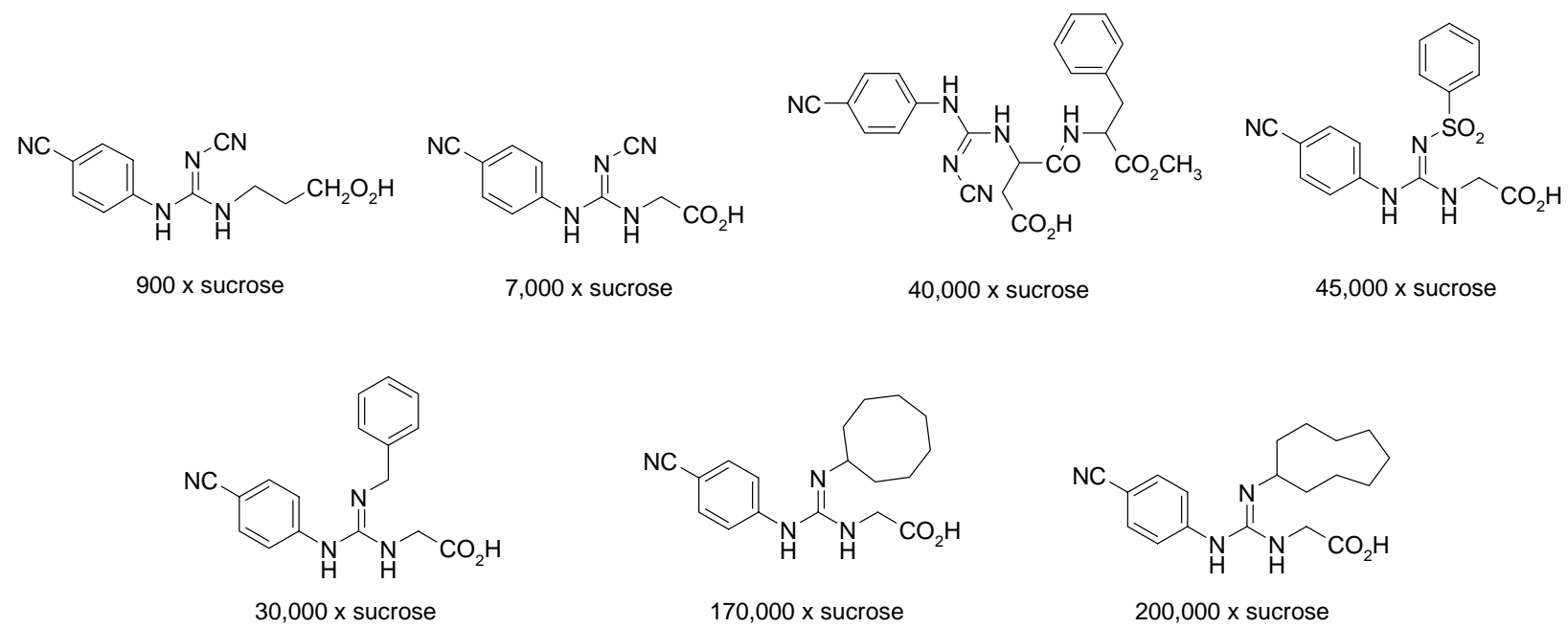

Figure 1. Superpotent guanidine sweeteners.

Guanidines are also known as useful basic catalysts. ${ }^{1 \mathrm{a}, 9}$ Several reports describe the synthesis of chiral guanidines and their use in asymmetric synthesis. ${ }^{10 a-c}$

An excellent review of preparative methods for guanidines was prepared by Anslyn et al., ${ }^{11 a}$ and others have emphasized solid phase synthesis. ${ }^{11 \mathrm{~b}, \mathrm{c}}$

Their wide importance has prompted the investigation of new approaches to guanidine derivatives. In this overview we summarize recent advances in the development of guanylating reagents (i.e. compounds which form the guanidine structure by a chemical transformation) for guanidine synthesis. Functionalizations of pre-existing guanidine cores, e.g., by alkylation, arylation or acylation are not covered. We do not attempt to catalog exhaustively the enormous 
range of synthetic guanidine derivatives, but limit our review to reports describing the discovery of guanylating reagents, and investigations of reaction conditions (time, temperature, catalysis etc.) undertaken to improve the yields of guanidines. Reports of the preparation of guanidine compounds by previously developed methods (such as the synthesis of guanidine natural products and oligomers) are also outside the scope of this report.

\section{Reagents for the preparation of guanidines}

\subsection{Thioureas}

Thioureas are common reagents for the synthesis of guanidines. Usually the conversion of a thiourea into a guanidine requires initial activation. However, in many cases the characterization, isolation or even definition of active intermediates is not described.

Scheme 1 presents the conversion of thioureas $\mathbf{2}$ into guanidines $\mathbf{4}$ in a suitable solvent (THF, acetonitrile, or chloroform) containing copper sulphate - silica gel in the presence of tertiary amines. $^{12}$ The formation of the intermediate carbodiimide 3 (non-isolable under these conditions) proceeds quickly and the overall reaction time is short (Table 1). The presence of a tertiary amine, e.g., triethylamine, accelerates the desulfurization. The procedure allows the preparation a very wide range of di-, tri- and tetra-substituted guanidines.

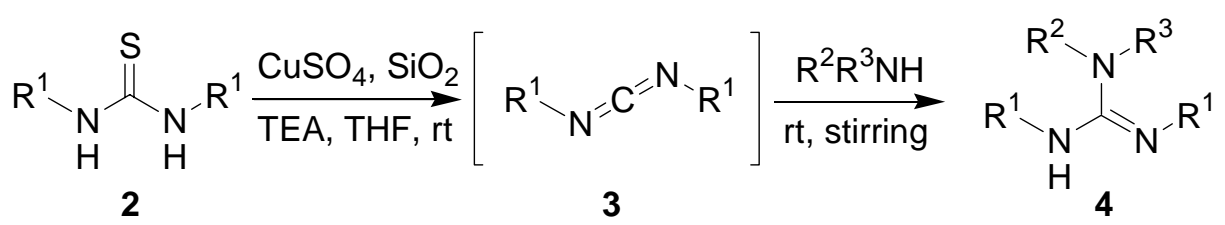

\section{Scheme 1}

Table 1. One pot desulfurization of thioureas and reaction with an amine by the procedure of Scheme 1

\begin{tabular}{cccccc}
\hline Entry & $\mathrm{R}^{1}$ & $\mathrm{R}^{2}$ & $\mathrm{R}^{3}$ & Reaction time, min & Yield, \% \\
\hline 1 & $\mathrm{Ph}$ & $\mathrm{CH}_{3}$ & $\mathrm{H}$ & 50 & 78 \\
2 & $\mathrm{Ph}$ & $\mathrm{CH}_{3}$ & $\mathrm{CH}_{3}$ & 55 & 75 \\
3 & $\mathrm{Ph}$ & $\mathrm{C}_{6} \mathrm{H}_{11}$ & $\mathrm{H}$ & 50 & 85 \\
4 & $\mathrm{Ph}$ & $\mathrm{C}_{2} \mathrm{H}_{5}$ & $\mathrm{C}_{2} \mathrm{H}_{5}$ & 50 & 90 \\
5 & $\mathrm{Ph}$ & $\mathrm{PhCH}_{2}$ & $\mathrm{H}$ & 50 & 80 \\
6 & $\mathrm{Ph}$ & $n-\mathrm{Bu}$ & $\mathrm{H}$ & 45 & 75 \\
7 & $o-\mathrm{Tolyl}$ & $\mathrm{C}_{2} \mathrm{H}_{4} \mathrm{OH}$ & $\mathrm{H}$ & 55 & 75 \\
8 & $\mathrm{Ph}$ & $\mathrm{H}$ & $\mathrm{H}$ & 50 & 90 \\
9 & $o-\mathrm{Tolyl}$ & $\mathrm{H}$ & $\mathrm{H}$ & 50 & 85 \\
10 & $\mathrm{C}_{6} \mathrm{H}_{11}$ & $\mathrm{H}$ & $\mathrm{H}$ & 60 & 86 \\
11 & $\mathrm{n}-\mathrm{Bu}$ & $\mathrm{H}$ & $\mathrm{H}$ & 50 & 80 \\
\hline
\end{tabular}


Electron withdrawing substituents in the thiourea fragment also accelerate the reaction. Many recent synthetic approaches utilize protected thioureas, containing electron withdrawing protecting groups which can be removed by standard methods. Thus, di-Boc protected thiourea 5 is converted into guanidines 7 by amines in the presence of 1-methyl-2-chloropyridinium iodide $^{13} 6$ (Mukaiyama's reagent, Scheme 2, Table 2) as follows: (i) primary and unhindered secondary amines are guanylated in high (>80\%) yield using a slight excess of reagent in anhydrous DMF (entries 1-4); (ii) for hindered or unreactive amines, methylene chloride provides a substantial increase in yield (entries 7 and 8) over reactions run in DMF (entries 5 and 7). The effect of solvent on yield probably results from the instability of the carbodiimide intermediates. When nucleophilic attack by an amine is slow, competitive decomposition of the carbodiimide occurs. In methylene chloride, the reactions are heterogeneous owing to sparing solubility of the di-Boc protected thiourea; it is believed that this results in slower production of the di-Boc carbodiimide and consequently, more efficient consumption of the thioureum by less reactive amines. The guanylation of resin-bound amines under these conditions was also examined.

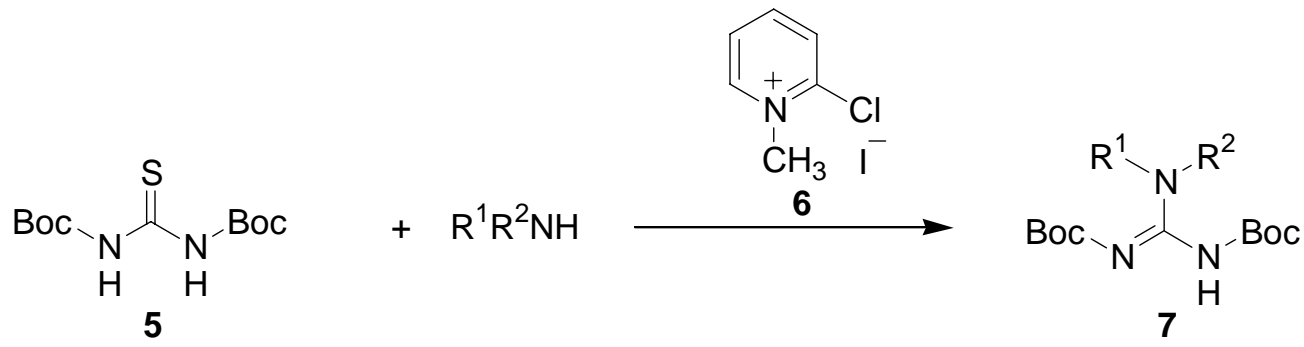

\section{Scheme 2}

Table 2. Preparation of guanidines from thioureas promoted by Mukaiyama's reagent (Scheme 2)

\begin{tabular}{cccc}
\hline Entry & $\mathrm{R}^{1} \mathrm{R}^{2} \mathrm{NH}$ & Solvent & Product yield, \% \\
\hline 1 & $\mathrm{PhCH}_{2} \mathrm{NH}_{2}$ & $\mathrm{DMF}$ & 91 \\
2 & Diallylamine & $\mathrm{DMF}$ & 86 \\
3 & Piperidine & $\mathrm{DMF}$ & 57 \\
4 & $\mathrm{DMF}_{\mathrm{CO}_{2} \mathrm{Me}}$ & & 85 \\
& & \\
5 & Diisopropylamine & $\mathrm{DMF}$ & 21 \\
6 & Diisopropylamine & $\mathrm{CH}_{2} \mathrm{Cl}_{2}$ & 71 \\
7 & Aniline & $\mathrm{DMF}^{2}$ & 34 \\
8 & Aniline & $\mathrm{CH}_{2} \mathrm{Cl}$ & 92 \\
9 & 4-Nitropyrazole & $\mathrm{DMF}_{2}$ & 43 \\
\hline
\end{tabular}


Ethoxycarbonyl substituted thioureas $\mathbf{8}$ are prepared using the corresponding isothiocyanate (Scheme 3). Guanidines 9 are prepared by displacement of sulfur in the presence of ethyl-3aminopropyl carbodiimide hydrochloride (EDCI) ${ }^{14}$ in $48 \mathrm{~h}$ without the formation of any major side products. The guanidines obtained via EDCI coupling (Table 3) were easily purified by flash chromatography. Deprotection can be achieved by the treatment with $\mathrm{Me}_{3} \mathrm{SiBr}$.<smiles>[R17]NC(=NC(=O)OCC)NCc1ccccc1</smiles>

\section{Scheme 3}

Table 3. One pot preparation of guanidines by EDCI desulfurization and reaction of with amines (of Scheme 3)

\begin{tabular}{clcclc}
\hline Entry & $\mathrm{R}^{1} \mathrm{NH}_{2}$ & Yield, \% & Entry & $\mathrm{R}^{1} \mathrm{NH}_{2}$ & Yield, \% \\
\hline 1 & Benzylamine & 80 & 6 & 2-Methylbenzylamine & 80 \\
2 & 4-Aminobenzylamine & 83 & 7 & Aniline & 87 \\
3 & 4-Nitrobenzylamine & 88 & 8 & $i$-Propylamine & 85 \\
4 & 4-Methoxybenzylamine & 85 & 9 & $t$-Butylamine & 83 \\
5 & 2,4-Dimethoxybenzylamine & 78 & & & \\
\hline
\end{tabular}

Amines can be converted into guanidines $\mathbf{1 1}$ by the reaction di-Boc thiourea $5^{15}$ in the presence of mercuric chloride or copper(II) chloride in the presence of triethylamine (Scheme 4). The influence of the desulphurizing agent is shown in Table 4.
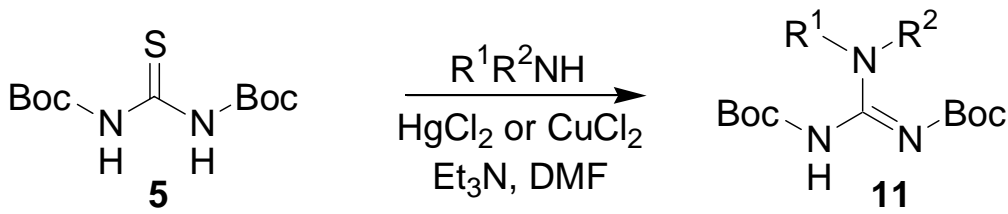

\section{Scheme 4}


Table 4. Preparation of guanidines in the presence of copper or mercuric salts (see Scheme 4)

\begin{tabular}{|c|c|c|c|c|}
\hline Entry & Amine & Metal Salt & Conditions & Yield, \% \\
\hline \multirow[t]{2}{*}{1} & & $\mathrm{HgCl}_{2}$ & $0{ }^{\circ} \mathrm{C}, 20 \mathrm{~min}$ & 90 \\
\hline & & $\mathrm{CuCl}_{2}$ & $0{ }^{\circ} \mathrm{C}, 0.5 \mathrm{~h}, \mathrm{rt}, 0.5 \mathrm{~h}$ & 62 \\
\hline 2 & & $\mathrm{HgCl}_{2}$ & $60{ }^{\circ} \mathrm{C}, 2 \mathrm{~h}$ & 87 \\
\hline \multirow[t]{2}{*}{3} & ${ }_{2} \mathrm{NH}_{2}$ & $\mathrm{HgCl}_{2}$ & rt. $1 \mathrm{~h}$ & 89 \\
\hline & & None & $60{ }^{\circ} \mathrm{C}, 4 \mathrm{~h}$ & 0 \\
\hline \multirow[t]{3}{*}{4} & & $\mathrm{HgCl}_{2}$ & $\mathrm{rt}, 2 \mathrm{~h}$ & 78 \\
\hline & $\mathrm{NH}_{2}$ & $\mathrm{HgCl}_{2}$ & $\mathrm{rt}, 20 \mathrm{~h}$ & 92 \\
\hline & & none & $60{ }^{\circ} \mathrm{C}, 6 \mathrm{~h}$ & 0 \\
\hline 5 & $\left(\left(\mathrm{CH}_{3}\right)_{2} \mathrm{CH}\right)_{2} \mathrm{NH}$ & $\mathrm{HgCl}_{2}$ & $0{ }^{\circ} \mathrm{C}, 0.5 \mathrm{~h}$ & 90 \\
\hline
\end{tabular}

$N$-Boc- $N$ '-alkyl or aryl thioureas 12 reacted smoothly with an aryl or alkyl amine in the presence of $\mathrm{HgCl}_{2}{ }^{16 \mathrm{a}}$ (Scheme 5). The $N$ - boc- $N^{\prime}, N^{\prime \prime}$-disubstituted guanidine products 13 are easily deprotected, as shown in Scheme 5, providing an efficient route to $N, N$ '-disubstituted guanidines14, which compares favorably with other methods (Table 5). For an adaptation to solid phase synthesis see ref $16^{\mathrm{b}}$.

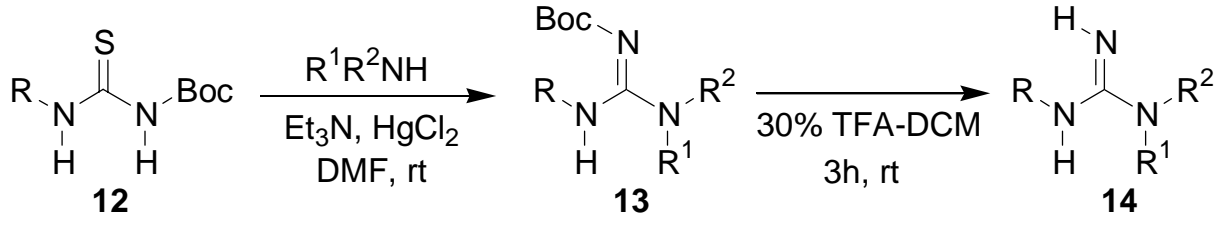

\section{Scheme 5}

Table 5. Synthesis of protected guanidines in the presence of $\mathrm{HgCl}_{2}$ (see Scheme 5)

\begin{tabular}{ccccc}
\hline Entry & $\mathrm{X}$ & $\mathrm{R}$ & $\mathrm{R}^{1} \mathrm{R}^{2} \mathrm{NH}$ & Yield, \% \\
\hline 1 & Boc & Cyclohexyl & 2-Tetrahydroisoquinoline & 63 \\
2 & Boc & Cyclohexyl & Aniline & 70 \\
3 & Boc & 4-Nitrophenyl & 4-Methoxyaniline & 85 \\
4 & $\mathrm{CH}_{3} \mathrm{CO}$ & Cyclohexyl & 2-Tetrahydroisoquinoline & 75 \\
5 & $\mathrm{PhCO}$ & Cyclohexyl & 2-Tetrahydroisoquinoline & 75 \\
6 & $\mathrm{PhCH}_{2} \mathrm{CO}$ & Cyclohexyl & 2-Tetrahydroisoquinoline & 67 \\
7 & Ts & Cyclohexyl & 2-Tetrahydroisoquinoline & $83\left(41^{\mathrm{a}}\right)$ \\
8 & $\mathrm{CN}$ & Cyclohexyl & 2-Tetrahydroisoquinoline & $61^{\mathrm{a}}$ \\
\hline
\end{tabular}

${ }^{a}$ Overall yield for the two steps: formation of thiourea and in situ conversion into a guanidine derivative. 
Carbamoyl isothiocyanates $\mathbf{1 5}$ possess several advantages for the preparation of thioureas ${ }^{17}$ (Scheme 6, Table 6): These reagents provide a protecting group throughout the synthesis, facilitating purification, and they show increased reactivity over alkyl isothiocyanates, forming thioureas even with hindered amines. A second amine can be coupled to the carbamoyl thiourea 16 using EDCI, forming 1,3-disubstituted and 1,1,3-trisubstituted guanidines 17 through either a stepwise or one-pot synthesis. To gauge the steric and electronic limitations of this procedure, amines of varying reactivity (A-G) were investigated for their ability to form protected thiourea and guanidine; yields for reactions of ethoxycarbonyl isothiocyanate are shown in Table 6. This procedure is general for other carbamoyl isothiocyanates (ethyl carbamate, benzyl carbamate (Cbz), 2,2,2-trichloro-1,1-dimethylethyl carbamate, fluorenylmethyl carbamate (Fmoc), and phenyl carbamate) which allows synthetic flexibility in the deprotection.

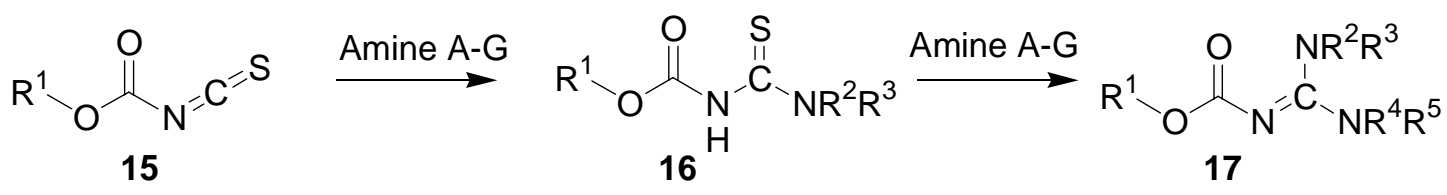<smiles>NCc1ccccc1</smiles>

A

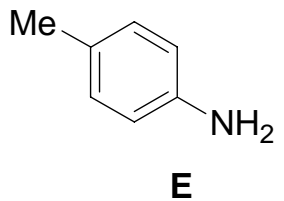

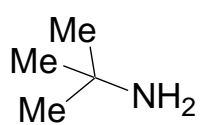

B<smiles>C1COCCN1</smiles>

C<smiles>CC(C)NC(C)C</smiles>

D

\section{Scheme 6}

Table 6. Synthesis of protected thioures 16 and guanidines 17 (see Scheme 6)

\begin{tabular}{ccccccccc}
\hline & & \multicolumn{7}{c}{ \% Yield of guanidine $\mathbf{1 7}$} \\
\hline Amine & \% Yield of thiourea 16 & A & B & C & D & E & F & G \\
A & 99 & 99 & 99 & 95 & 76 & 92 & 82 & 85 \\
B & 99 & 99 & 74 & 59 & 55 & 99 & 99 & 65 \\
C & 99 & 0 & 0 & 0 & 0 & 0 & 0 & 0 \\
D & 99 & 0 & 0 & 0 & 0 & 0 & 0 & 0 \\
E & 92 & 99 & 87 & 99 & 81 & 95 & 97 & 34 \\
F & 77 & 96 & 99 & 61 & 84 & 98 & 67 & 39 \\
G & 72 & 85 & 76 & 99 & 42 & 35 & 34 & 0 \\
\hline
\end{tabular}


$N$-Hydroxyguanidines 19 were prepared from 1-benzyloxy-3-Cbz-thiourea $\mathbf{1 8}^{18}$ (Scheme 7, Table 7). The Cbz strategy was chosen because cleavage of the Cbz and benzyl groups could be accomplished simultaneously. The solvent of choice was DMF; mercuric chloride provided efficient desulphurization.
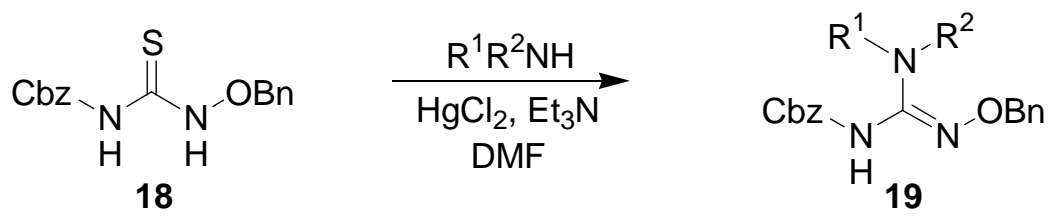

\section{Scheme 7}

Table 7. Synthesis of hydroxyguanidines in the presence of $\mathrm{HgCl}_{2}$ (see Scheme 7)

\begin{tabular}{cccc}
\hline Entry & Amine & Reaction time, h & Yield, \% \\
\hline 1 & Benzyl amine & 7 & 47 \\
2 & 2-Aminocarbonylaziridine & 9 & 34 \\
3 & Aniline & 7.5 & 54 \\
4 & Diisopropylamine & 9 & 34 \\
5 & 3-Hydroxypropylamine & 7 & 35 \\
6 & Aminoadamantane & 10.5 & 67 \\
\hline
\end{tabular}

The desulphurization of a thiourea to a carbodiimide and subsequent reaction with an amine was applied to solid phase synthesis. ${ }^{19}$ The thiourea 20 was treated with an amine (5 equiv.) at $50^{\circ} \mathrm{C}$ in $\mathrm{CHCl}_{3}$ in the presence of DIC (diisopropylcarbodiimide) (5 equiv.) and DIEA (5 equiv.) to give the resin bound guanidine 21. The disubstituted guanidine 22 was then cleaved under mild Rink resin cleavage conditions (25\% TFA/ $\mathrm{CH}_{2} \mathrm{Cl}_{2}$ at room temperature) (Scheme 8, Table 8).

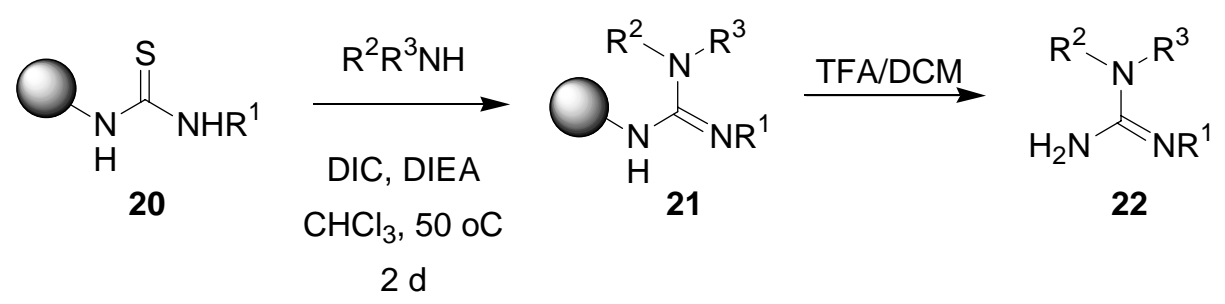

\section{Scheme 8}


Table 8. Conversion of thioureas to guanidines on solid phase (of Scheme 8)

\begin{tabular}{|c|c|c|c|}
\hline Entry & Product & Yield, \% & Purity \\
\hline 1 & & 71 & 70 \\
\hline 2 & & 77 & 54 \\
\hline 3 & & 70 & 56 \\
\hline 4 & & 95 & 86 \\
\hline 5 & & 98 & 91 \\
\hline 6 & & 89 & 75 \\
\hline 7 & & 87 & 90 \\
\hline 8 & & 95 & 85 \\
\hline 9 & & 96 & 95 \\
\hline 10 & & 100 & 83 \\
\hline 11 & & 99 & 83 \\
\hline 12 & & 88 & 83 \\
\hline
\end{tabular}

The approach of Scheme 9 combines the advantages of traditional solution phase chemistry with the application of polymeric reagents. ${ }^{20}$ The desired compounds are obtained in a high throughput manner without additional purification, and in satisfactory purity. No base is required.

\section{Scheme 9}

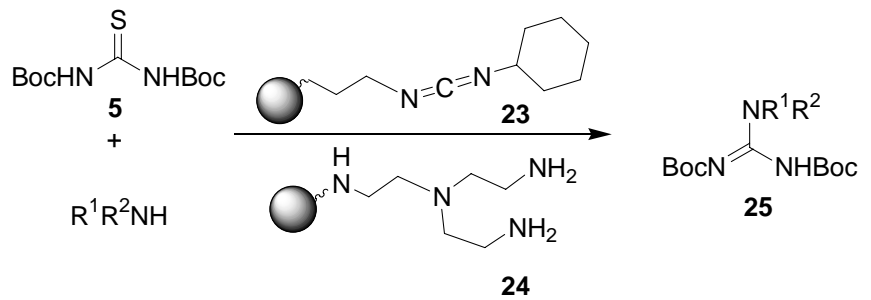


The use of TFA-cleavable Pbf-group protection/activation is an advantageous alternative for the synthesis of guanidines ${ }^{21 a}$ (Scheme 10). Primary or secondary amines, including tertbutylamine and diisopropylamine, in the presence of Mukaiyama's reagent, give guanidines 27 in high yields at room temperature and in 12-18 h. The high efficiency of Mukaiyama's reagent in promoting the guanidinylating reaction contrasts with its role in sulfamoylthiourea based systems. The transformation also succeeds with EDCI in place of Mukaiyama's reagent. No heavy metal salt or excessive heating was needed.

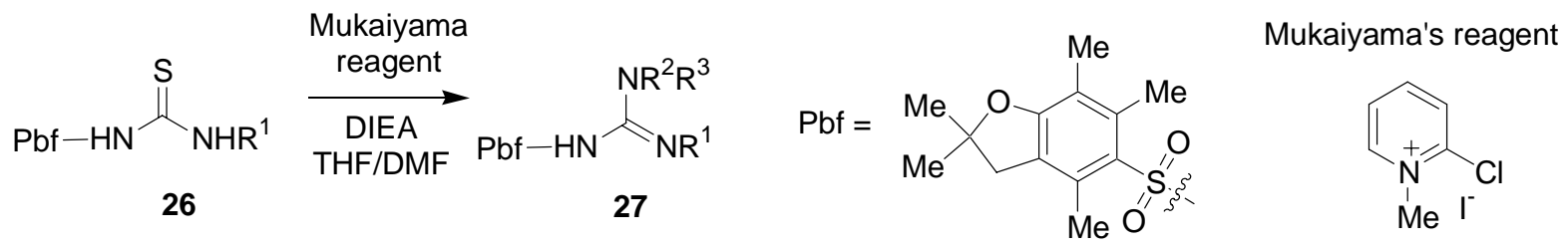

\section{Scheme 10}

Solid phase strategy for the preparation of guanidines $\mathbf{3 0}$ has also applied triphenylphosphine dichloride as a desulphurizing agent. ${ }^{21 b}$ The immobilised thiourea 28 was treated with triphenylphosphine dichloride freshly prepared from triphenylphosphine with hexachloroethane in THF. The use of base proved to be detrimental.

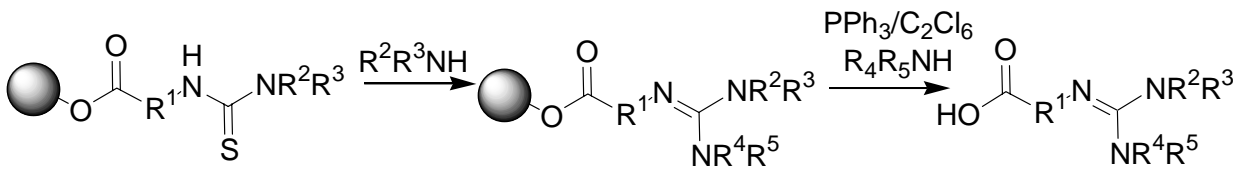

\section{Scheme 11}

The preparation of $N, N^{\prime}$-disubstituted acylguanidines from primary amides 31, isothiocyanates 32 and amines ${ }^{21 \mathrm{~b}}$ has utilized three alternative one pot procedures: $\mathrm{HgCl}_{2}$, EDCI and Mukaiyama’s reagent; each showed comparable yields of guanidines.

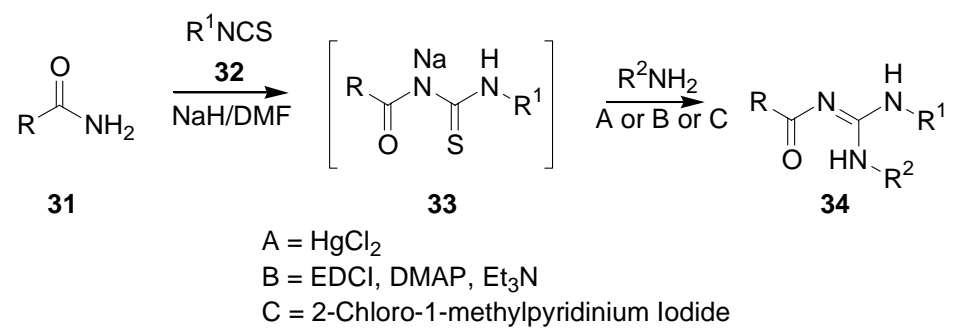

\section{Scheme 12}

\subsection{Isothioureas}

As well as thioureas, isothioureas, particularly $S$-methylisothioureas, are well developed as guanylating agents due to their easy preparation and availability. 
Guanidines have been successfully prepared from $N$-arylsulfonyl $S$-methylisothioureas. ${ }^{22}$ The Mtr-reagent 35 (Mtr is 4-methoxy-2,3,6-trimethylphenylsulfonyl) reacted with piperidine or aniline in the presence of triethylamine and $\mathrm{Hg}\left(\mathrm{ClO}_{4}\right)_{2}$ in refluxing THF (or toluene) to produce mono Mtr-protected guanidines 36 in moderate to good yields (Schemes 13, Table 10). Higher yields were obtained when the reactions were carried out in refluxing THF in the presence of triethylamine and mercuric perchlorate.

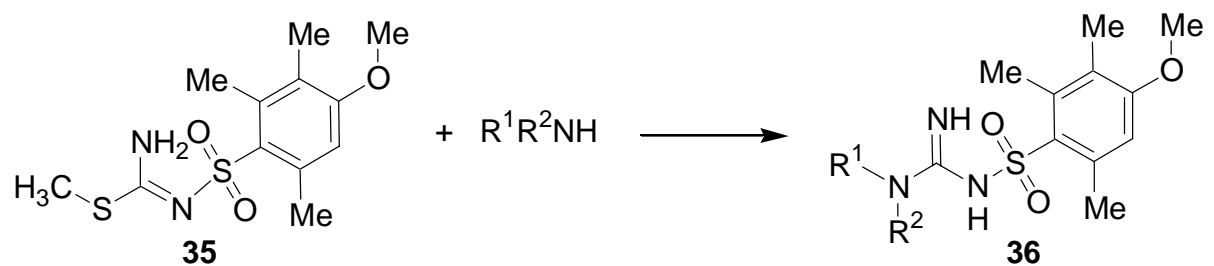

\section{Scheme 13}

Table 10. Synthesis of Mtr-protected guanidines 36 (of Scheme 13)

\begin{tabular}{|c|c|c|c|}
\hline Entry & $\mathrm{R}^{1} \mathrm{R}^{2} \mathrm{NH}$ & Conditions & Yield, \% \\
\hline 1 & & $\mathrm{HgCl}_{2}$ (1.1 eq.), THF, $\Delta$ & 42 \\
\hline 2 & & $\begin{array}{l}\mathrm{HgCl}_{2}\left(1.1 \text { eq.), } \mathrm{Et}_{3} \mathrm{~N} \text { (2 eq.), }\right. \\
\text { toluene, } \Delta\end{array}$ & 37 \\
\hline 3 & & $\begin{array}{c}\mathrm{Hg}\left(\mathrm{ClO}_{4}\right)_{2}(1.1 \text { eq. }), \mathrm{Et}_{3} \mathrm{~N} \text { (2 eq.), } \\
\text { toluene, } \Delta\end{array}$ & 62 \\
\hline 4 & & $\begin{array}{c}\mathrm{AgClO}_{4}(1.1 \text { eq. }), \mathrm{Et}_{3} \mathrm{~N} \text { (2 eq.), } \\
\text { toluene, } \Delta\end{array}$ & 46 \\
\hline 5 & & $\begin{array}{c}\mathrm{Hg}\left(\mathrm{ClO}_{4}\right)_{2}(1.1 \text { eq. }), \mathrm{Et}_{3} \mathrm{~N} \text { (2 eq.), } \\
\text { THF, } \Delta\end{array}$ & 80 \\
\hline 6 & & $\begin{array}{l}\mathrm{HgCl}_{2} \text { (1.1 eq.), } \mathrm{Et}_{3} \mathrm{~N} \text { (2 eq.), } \\
\text { toluene, } \Delta\end{array}$ & $>20$ \\
\hline 7 & & $\begin{array}{c}\mathrm{Hg}\left(\mathrm{ClO}_{4}\right)_{2}(1.1 \mathrm{eq} .), \mathrm{Et}_{3} \mathrm{~N} \text { (2 eq.), } \\
\text { toluene, } \Delta\end{array}$ & 47 \\
\hline 8 & & $\begin{array}{c}\mathrm{HgCl}_{2} \text { (1.1 eq.), } \mathrm{Et}_{3} \mathrm{~N} \text { (1.5 eq.), } \\
\text { THF, } \Delta\end{array}$ & 51 \\
\hline 9 & & $\begin{array}{c}\mathrm{AgClO}_{4}\left(1.1 \text { eq.), } \mathrm{Et}_{3} \mathrm{~N} \text { (2 eq.), }\right. \\
\text { toluene, } \Delta\end{array}$ & 71 \\
\hline 10 & & $\begin{array}{c}\mathrm{Hg}\left(\mathrm{ClO}_{4}\right)_{2}(1.1 \text { eq. }), \mathrm{Et}_{3} \mathrm{~N} \text { (2 eq.), } \\
\mathrm{THF}, \Delta\end{array}$ & 93 \\
\hline
\end{tabular}


Reagents 35 and 37 (Schemes 13,14) were evaluated with several substrates to determine optimal conditions (Tables 10,11). The reagents were reacted with Boc-Lys-OMe.HC1 and Bocp-aminophenylalanine-OMe. The protected lysine ester (Table 11, Entries 1-3) has a primary aliphatic amine side chain but appears to react with $\mathbf{3 5}$ and $\mathbf{3 7}$ less readily and in lower yield, than the other substrates. The Boc-p-aminophenylalanine-OMe substrate reacts efficiently with both reagents in high yield to produce the target guanylated amino acids (Entry 4). These results indicate that arylsulfonyl isothioureas react with the less nucleophilic electron deficient amines more readily than the more basic primary aliphatic amines.<smiles>CSC(=N)NS(=O)(=O)c1c(C)c(C)c2c(c1C)CCC(C)(C)O2</smiles>

37

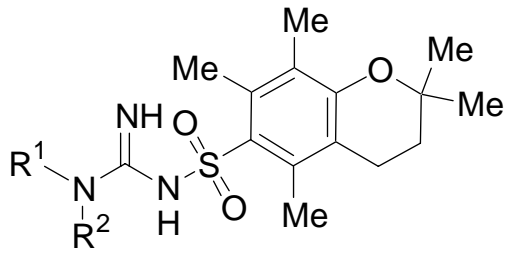

38

\section{Scheme 14}

Table 11. Synthesis of Pbf-protected guanidines 36 by procedure of Scheme 14

\begin{tabular}{lll}
\hline Entry & \multicolumn{1}{c}{ Conditions } & Yield, \% \\
\hline 1 & $\mathrm{Hg}\left(\mathrm{ClO}_{4}\right)_{2}\left(1.1\right.$ eq.), $\mathrm{Et}{ }_{3} \mathrm{~N}$ (2 eq.), toluene, $\Delta, 16 \mathrm{~h}$ & 24 \\
\hline
\end{tabular}

The relatively easy activation of thioureas 39 as thiazetidines 40 provides tri- and tetrasubstituted guanidines $\mathbf{4 1}{ }^{23}$ (Scheme 15, Table 12) in good to excellent yields.

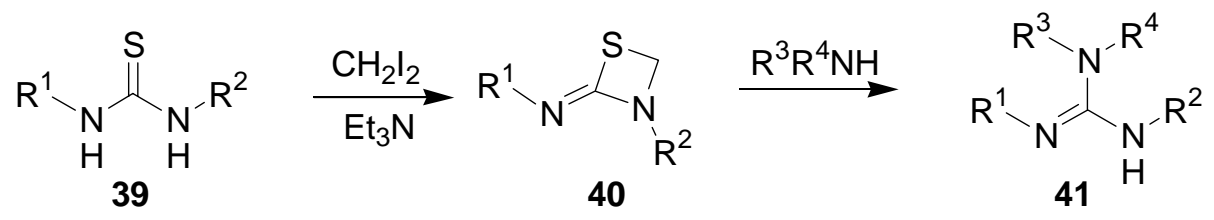

Scheme 15 
Table 12. Synthesis of polysubstituted guanidines via thiazetidine derivatives $\mathbf{4 0}$ (of Scheme 15)

\begin{tabular}{lllllc}
\hline Entry & $\mathrm{R}^{1}$ & $\mathrm{R}^{2}$ & $\mathrm{R}^{3}$ & $\mathrm{R}^{4}$ & Yield,\% \\
\hline 1 & $2,4-\mathrm{Cl}_{2}-\mathrm{C}_{6} \mathrm{H}_{3}-\mathrm{CO}$ & $4-\mathrm{Cl}-\mathrm{C}_{6} \mathrm{H}_{4}$ & $\mathrm{PhCH}_{2}$ & $\mathrm{H}$ & 92 \\
2 & $2,4-\mathrm{Cl}_{2}-\mathrm{C}_{6} \mathrm{H}_{3}-\mathrm{CO}$ & $4-\mathrm{Cl}-\mathrm{C}_{6} \mathrm{H}_{4}$ & $\mathrm{CH}_{3}$ & $\mathrm{H}$ & 68 \\
3 & $2,4-\mathrm{Cl}_{2}-\mathrm{C}_{6} \mathrm{H}_{3}-\mathrm{CO}$ & $4-\mathrm{Cl}-\mathrm{C}_{6} \mathrm{H}_{4}$ & $n-\mathrm{C}_{3} \mathrm{H}_{7}$ & $\mathrm{H}$ & 90 \\
4 & $2,4-\mathrm{Cl}_{2}-\mathrm{C}_{6} \mathrm{H}_{3}-\mathrm{CO}$ & $4-\mathrm{Cl}-\mathrm{C}_{6} \mathrm{H}_{4}$ & $i-\mathrm{C}_{3} \mathrm{H}_{7}$ & $\mathrm{H}$ & 89 \\
5 & $2,4-\mathrm{Cl}_{2}-\mathrm{C}_{6} \mathrm{H}_{3}-\mathrm{CO}$ & $4-\mathrm{Cl}-\mathrm{C}_{6} \mathrm{H}_{4}$ & $\mathrm{CH}_{3}$ & $\mathrm{CH}_{3}$ & 80 \\
6 & $2,4-\mathrm{Cl}_{2}-\mathrm{C}_{6} \mathrm{H}_{3}-\mathrm{CO}$ & $4-\mathrm{Cl}-\mathrm{C}_{6} \mathrm{H}_{4}$ & $(\mathrm{EtO})_{2} \mathrm{CHCH}_{2}$ & $\mathrm{H}$ & 58 \\
7 & $4-\mathrm{CH}_{3}-\mathrm{C}_{6} \mathrm{H}_{4}-\mathrm{CO}$ & $\mathrm{Ph}$ & $\mathrm{CH}_{3}$ & $\mathrm{CH}_{3}$ & 99 \\
8 & $4-\mathrm{CH}_{3}-\mathrm{C}_{6} \mathrm{H}_{4}-\mathrm{CO}$ & $\mathrm{Ph}$ & $\left(\mathrm{CH}_{2}\right)_{5}$ & & 76 \\
9 & $4-\mathrm{CH}_{3}-\mathrm{C}_{6} \mathrm{H}_{4}-\mathrm{CO}$ & $\mathrm{Ph}$ & $\left(\mathrm{CH}_{2}\right)_{2} \mathrm{O}\left(\mathrm{CH}_{2}\right)_{2}$ & & 88 \\
\hline
\end{tabular}

Reactions of S-methylisothioureas $\mathbf{4 2}{ }^{24}$ with various cyclic amines in refluxing tert-butyl alcohol gave almost a hundred salts of guanidine (Table 13, See Supplemental Materials) in fair to good yields (Scheme 16), accommodating phenyl substituents ranging from strongly electron withdrawing to strongly electron donating, as well as those with bulky substituents in the orthoposition. Either the isothiourea or the amine should be in the form of a soluble salt to achieve the satisfactory reaction rates.

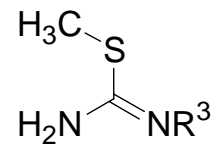

42

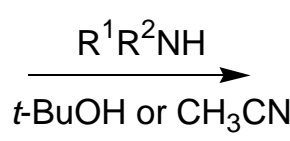

$\mathrm{H}_{2} \mathrm{~N} \mathrm{NR}^{3}$

43

\section{Scheme 16}

Mild and efficient promotion by mercuric chloride converts di-Cbz-isothioureas $\mathbf{4 4}$ into protected guanidines $\mathbf{4 5}^{25}$ (Scheme 17, Table 14). Free and Cbz-protected guanidinoacids 47 were prepared similarly (Scheme 18, Table 15) from $\mathbf{4 4}$ and $\mathbf{4 6}$ by in situ carboxyl protection with trimethylsilyl chloride at the first stage of the reaction. ${ }^{26}$ Simple crystallization after work up provided pure materials. The utility of generating a protected guanidine was established further by converting Gly-Gly to $\alpha$-(bisbenzyloxycarbonyl)guanidinoacetylglycine.

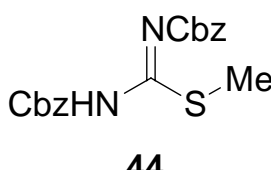

44

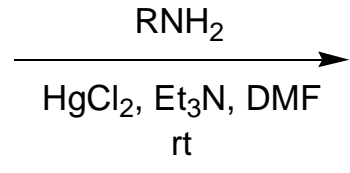

t

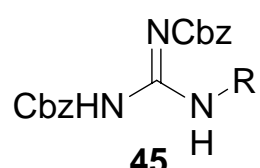

45

\section{Scheme 17}


Table 14. Synthesis of Cbz-substituted guanidines in the presence of $\mathrm{HgCl}_{2}$ (of Scheme 17)

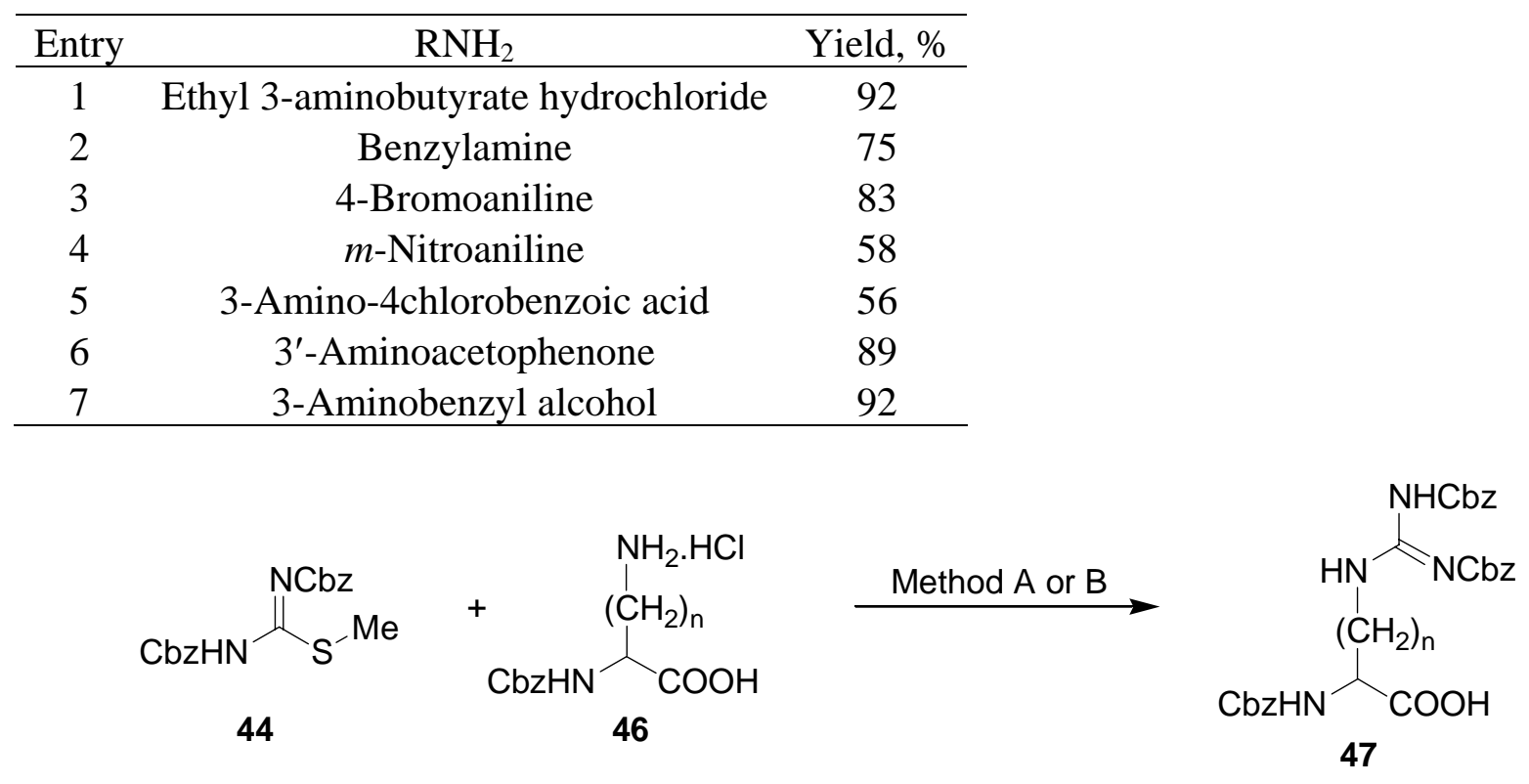

\section{Scheme 18}

Table 15. Synthesis of Cbz-substituted guanidines in the presence of $\mathrm{HgCl}_{2}$ (of Scheme 18)

\begin{tabular}{|c|c|c|c|c|}
\hline Entry & Amino acid & Method & $\begin{array}{c}\text { Molar ratio } \\
\text { Amino acid/reagent }\end{array}$ & Yield, \% \\
\hline 1 & $\mathrm{~N}^{\alpha}$-Cbz-L-Orn.HCl & A & $1: 1.25$ & 87 \\
\hline 2 & $\mathrm{~N}^{\alpha}$-Cbz-L-Lys.HCl & A & $1: 1.1$ & 62 \\
\hline 3 & $\mathrm{H}_{2} \mathrm{NCH}_{2} \mathrm{COOH}$ & B & $1.2: 1$ & 97 \\
\hline 4 & $(\mathrm{~S}) \mathrm{PhCH}_{2} \mathrm{CH}\left(\mathrm{NH}_{2}\right) \mathrm{COOH}$ & B & $1.2: 1$ & 84 \\
\hline 5 & $\mathrm{H}_{2} \mathrm{~N}\left(\mathrm{CH}_{2}\right)_{2} \mathrm{COOH}$ & B & $1.2: 1$ & 92 \\
\hline $6 a$ & $\mathrm{H}_{2} \mathrm{~N}\left(\mathrm{CH}_{2}\right)_{3} \mathrm{COOH}$ & B & $1.2: 1$ & 92 \\
\hline $6 b$ & $\mathrm{H}_{2} \mathrm{~N}\left(\mathrm{CH}_{2}\right)_{3} \mathrm{COOH} . \mathrm{HCl}$ & A & $1.2: 1$ & 92 \\
\hline 7 & $\mathrm{H}_{2} \mathrm{~N}\left(\mathrm{CH}_{2}\right)_{4} \mathrm{COOH}$ & B & $1.2: 1$ & 91 \\
\hline 8 & $\mathrm{H}_{2} \mathrm{~N}\left(\mathrm{CH}_{2}\right)_{5} \mathrm{COOH}$ & B & $1.2: 1$ & 80 \\
\hline 9 & HCl.p- $\mathrm{NH}_{2} \mathrm{CH}_{2} \mathrm{C}_{6} \mathrm{H}_{4} \mathrm{COOH}$ & A & $1.2: 1$ & 97 \\
\hline 10 & Gly-Gly & B & $1.2: 1$ & 69 \\
\hline
\end{tabular}

Guanidinoureas 49 and 50 were obtained by condensation of $N$-Cbz-ureido- $N$-Cbz-Smethylisothiourea $48^{27}$ with amines in the presence of triethyl amine in DMF at $20{ }^{\circ} \mathrm{C}$ (Scheme 19. Table 16). In the reaction with butylamine, a triazinedione by-product $\mathbf{5 2}$ formed, but all the other cases gave exclusively guanidine compounds. 


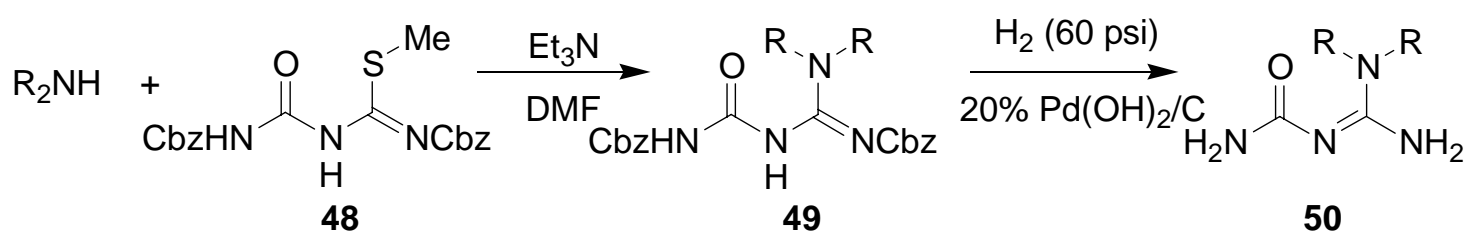

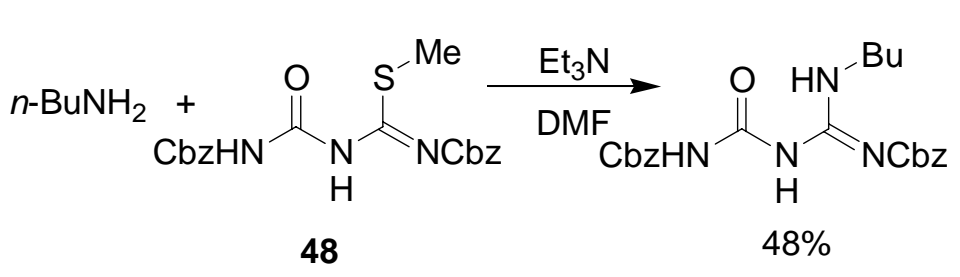

51<smiles>CC(C)(C)Nc1nc(=O)[nH]c(=O)n1CBr</smiles>

52

\section{Scheme 19}

Table 16. Synthesis of guanylureas 50

\begin{tabular}{cccc}
\hline Entry & Amine & Yield of protected product, \% & Yield of final product, \% \\
\hline 1 & Piperidine & 81 & 96 \\
2 & Morpholine & 75 & 97 \\
3 & $c$-Hexylamine & 99 & 93 \\
4 & $n$-Butylamine & 48 & 99 \\
\hline
\end{tabular}

A library of acylguanidinoureas has been reported. ${ }^{28 a}$ Isothioureas 53 were carbamoylated by a resin bound reagent, then acylated. Susequent aminolysis of the thiomethyl group in the presence of mercuric chloride led to $\mathbf{5 4}$ which were cleaved to give the guanidine library (Scheme 20, Table 17).<smiles>[R]C(=O)/N=C(\S)NC(=O)NC([R])C(=O)OCC</smiles>

53

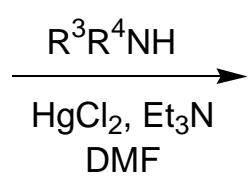

DMF<smiles>[R]C1=[R2]N([R])C(NC(=O)NC([R])C(=O)Oc2ccccc2)=N[R]1[H]</smiles>

54

\section{Scheme 20}


Table 17. Solid phase synthesis of guanylureas $\mathbf{5 4}$ (of Scheme 20)

\begin{tabular}{|c|c|c|c|c|c|c|}
\hline Entry & $\mathrm{R}^{1}$ & $\mathrm{R}^{2}$ & $\mathrm{R}^{3}$ & $\mathrm{R}^{4}$ & Yield,\% & Purity \\
\hline 1 & $\mathrm{H}$ & PhCH:CH & Benzyl & $\mathrm{H}$ & 55 & $72-95$ \\
\hline 2 & $\mathrm{CH}_{3}$ & $c$-Hexyl & Benzyl & $\mathrm{H}$ & 49 & $72-95$ \\
\hline 3 & i-Butyl & $\mathrm{PhCH}_{2}$ & 2-Benzothiazolyl & $\mathrm{H}$ & 83 & 72-95 \\
\hline 4 & $\mathrm{H}$ & $\mathrm{Ph}$ & $n$-Butyl & $\mathrm{H}$ & 91 & 85 \\
\hline 5 & $\mathrm{H}$ & $c$-Propyl & $2-\mathrm{CH}_{3} \mathrm{OC}_{6} \mathrm{H}_{4} \mathrm{CH}_{2}$ & $\mathrm{H}$ & 86 & 95 \\
\hline 6 & $\mathrm{H}$ & $2-\mathrm{NO}_{2} \mathrm{C}_{6} \mathrm{H}_{4}$ & i-Propyl & $i$-Propyl & 76 & 90 \\
\hline 7 & $\mathrm{H}$ & $2-\mathrm{CH}_{3} \mathrm{OC}_{6} \mathrm{H}_{4}$ & \multicolumn{2}{|l|}{$-\left(\mathrm{CH}_{2}\right)_{4^{-}}$} & 47 & 95 \\
\hline 8 & $i$-Butyl & $\mathrm{PhOCH}_{2}$ & Benzyl & $\mathrm{H}$ & 85 & 95 \\
\hline 9 & i-Butyl & $3-\mathrm{BrC}_{6} \mathrm{H}_{4}$ & Benzyl & $\mathrm{H}$ & 76 & 88 \\
\hline 10 & i-Butyl & 4-pyridyl & \multicolumn{2}{|l|}{$-\left(\mathrm{CH}_{2}\right)_{4^{-}}$} & 59 & 95 \\
\hline 11 & Benzyl & c-Hexyl & Benzyl & $\mathrm{H}$ & 93 & 57 \\
\hline 12 & Benzyl & $2-\mathrm{NO}_{2} \mathrm{C}_{6} \mathrm{H}_{4}$ & \multicolumn{2}{|l|}{$-\left(\mathrm{CH}_{2}\right)_{4^{-}}$} & 57 & 97 \\
\hline 13 & Benzyl & c-Propyl & Benzyl & $\mathrm{H}$ & 93 & 99 \\
\hline 14 & 4-Aminobutyl & $\mathrm{PhOCH}_{2}$ & Benzyl & $\mathrm{H}$ & 92 & 92 \\
\hline 15 & 4-Aminobutyl & $4-\mathrm{CH}_{3} \mathrm{OC}_{6} \mathrm{H}_{4} \mathrm{CH}_{2}$ & $2-\mathrm{CF}_{3} \mathrm{C}_{6} \mathrm{H}_{4}$ & $\mathrm{H}$ & 53 & 94 \\
\hline
\end{tabular}

Another solid support approach for acyl guanidine synthesis utilizes resin bound $S$ methylthioureas $55{ }^{28 \mathrm{~b}}$ Displacements of the methylthio group with ammonia, primary or secondary amines and aniline all proceeded cleanly at room temperature to give $\mathbf{5 6}$ which we than cleaved to 57.

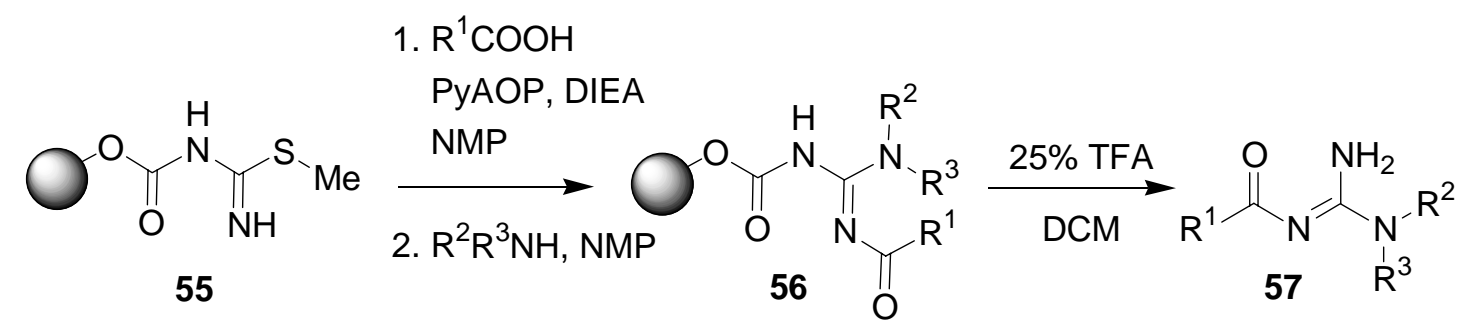

\section{Scheme 21}

Commercially available di-Boc-S-methylisothiourea $\mathbf{5 8}$ reacted with amines in the presence of mercuric chloride at $0-20{ }^{\circ} \mathrm{C}$ affording, after simple work up, the guanylated products $\mathbf{5 9}$ in goods yields ${ }^{29}$ (Scheme 22). The results, summarized in Table 18, illustrate the broad application of the reagent. Since the synthesis is equally successful with aliphatic and aromatic amines. Sterically hindered amines react well as do anilines with electron donating groups. Electron deficient anilines react slowly to afford the guanylated product in acceptable yields. 


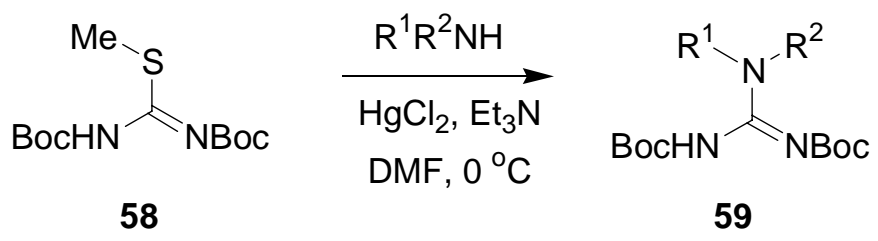

\section{Scheme 22}

Table 18. Preparation of guanidines 59

\begin{tabular}{cccc}
\hline Entry & $\mathrm{R}^{1}$ & $\mathrm{R}^{2}$ & Yield, \% \\
\hline 1 & $\mathrm{i}-\mathrm{Pr}$ & $\mathrm{i}-\mathrm{Pr}$ & 77 \\
2 & $\mathrm{Ph}$ & $\mathrm{H}$ & 89 \\
3 & 3- $\mathrm{HOCH}_{2} \mathrm{C}_{6} \mathrm{H}_{4}$ & $\mathrm{H}$ & 89 \\
4 & 3- $\mathrm{NO}_{2} \mathrm{C}_{6} \mathrm{H}_{4}$ & $\mathrm{H}$ & 68 \\
5 & - $^{-} \mathrm{HOC}_{6} \mathrm{H}_{4}$ & $\mathrm{H}$ & 87 \\
6 & 2- $\mathrm{NO}_{2}-4-\mathrm{HOC}_{6} \mathrm{H}_{4}$ & $\mathrm{H}$ & 87 \\
7 & o-Phenylenediamine & $\mathrm{H}, \mathrm{H}$ & 80 \\
\hline
\end{tabular}

Guanidinoglycosides 61 were prepared by the intramolecular cyclization of $\beta$-amino $N$ Fmoc-protected acyl isothioureas 60 (Scheme 23). A catalytic amount of DBU (0.7\% equiv) is optimal for converting these isothioureas into guanidinoglycosides (35-66\%) within an hour.
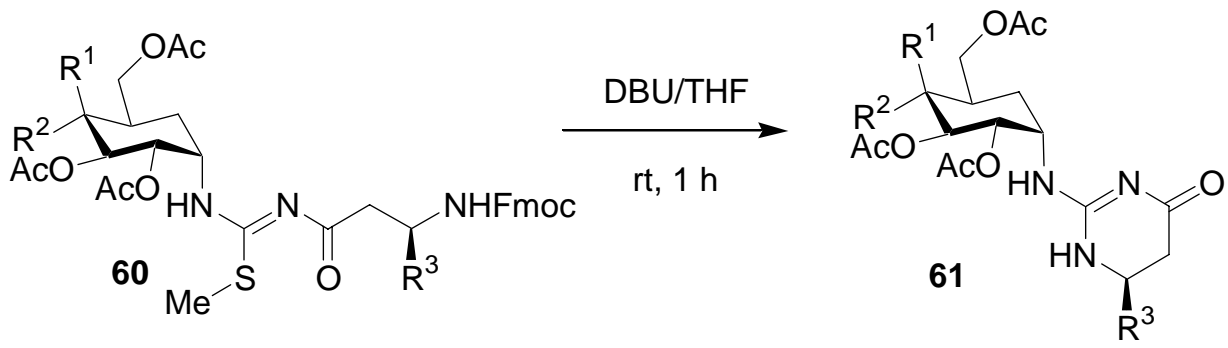

\section{Scheme 23}

S-Linked isothioureas 64 were formed via bis-electrophilic chlorothioformimines 63, as key intermediates. ${ }^{31}$ Dithiocarbamates were prepared from amines, carbon disulfide and benzyl chloride. Benzyl chloride was chosen for this study because it mimics the Merrifield resin. The dithiocarbamates 62 are quantitatively converted into the corresponding chlorothioformimines 63 by treatment at $60{ }^{\circ} \mathrm{C}$ with phosgene in toluene for $12 \mathrm{~h}$. The first amine converts chlorothioformimines 63 into the isothiourea 64 without double addition. The second substitution to give 65 was effected at $100{ }^{\circ} \mathrm{C}$, with an excess of a third amine, optimally in toluene. The reported reaction sequence is well adapted for SPS since it allows, in a four-step process, the addition of three primary amines under reaction conditions compatible with a wide variety of functional groups (Scheme 24). Moreover since the reaction conditions are suitable for automation and high-throughput synthesis, it appears possible to prepare large libraries of guanidines by this traceless linker strategy. 


\section{Scheme 24}

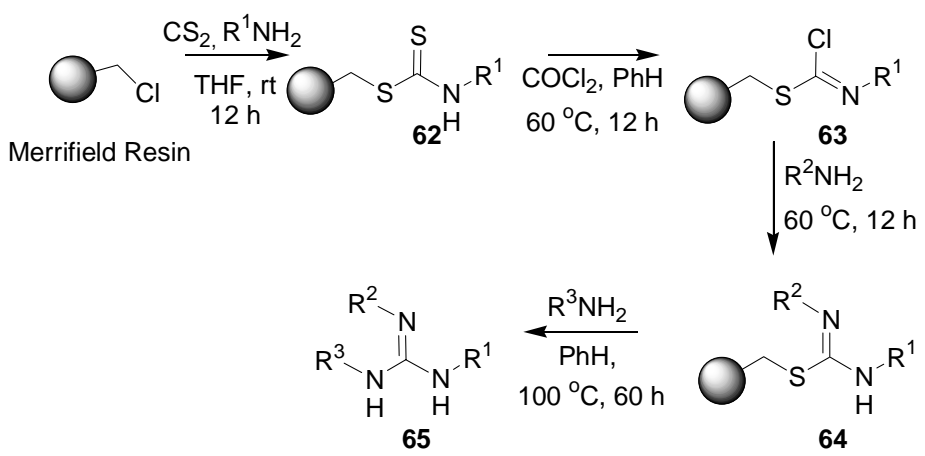

S-Linked isothioureas $\mathbf{6 6}$ as a masked guanidine scaffold allow the parallel synthesis of mono and dialkylated guanidines in high yield and purity ${ }^{32}$ (Scheme 25, Table 19). This procedure allows a high level of diversity using parallel array or combinatorial synthesis. The initial Mitsunobu step allows the use of either primary or secondary alcohols to generate $\mathbf{6 7}$ with the first point of diversity. Subsequent treatment of the resin bound $N$-alkyl isothioureas 67 with ammonia or primary amines liberates traceless guanidines $\mathbf{6 8}$ with a second point of diversity.

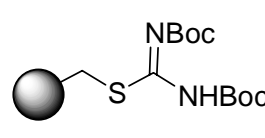

66

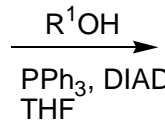

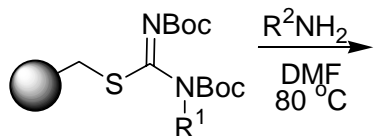

67

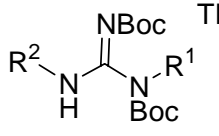

68<smiles>[R]NC(=N)N[R]C1CCCC1</smiles>

69

\section{Scheme 25}

Table 19. Traceless solid phase synthesis of guanidines via S-linked isothioureas (of Scheme 25)

\begin{tabular}{|c|c|c|c|c|}
\hline Entry & $\mathrm{R}^{1} \mathrm{OH}$ & $\mathrm{R}^{2} \mathrm{NH}_{2}$ & $\begin{array}{l}\text { Purity of } \\
\text { DiBoc, \% }\end{array}$ & $\begin{array}{c}\text { Yield of } \\
\text { DiBoc, \% }\end{array}$ \\
\hline 1 & $\mathrm{PhCH}_{2} \mathrm{OH}$ & $\mathrm{NH}_{3}$ & 95 & 88 \\
\hline 2 & $\mathrm{PhOCH}_{2} \mathrm{CH}_{2} \mathrm{OH}$ & $\mathrm{NH}_{3}$ & 95 & 100 \\
\hline 3 & $\mathrm{BocNH}\left(\mathrm{CH}_{2}\right)_{3} \mathrm{OH}$ & $\mathrm{NH}_{3}$ & 95 & 88 \\
\hline 4 & $\mathrm{PhCHC}\left(\mathrm{CH}_{3}\right) \mathrm{CH}_{2} \mathrm{OH}$ & $\mathrm{NH}_{3}$ & 95 & 95 \\
\hline 5 & $\left(\mathrm{CH}_{3}\right)_{2} \mathrm{CCHCH}_{2} \mathrm{OH}$ & $\mathrm{NH}_{3}$ & 100 & 95 \\
\hline 6 & $\left(\mathrm{CH}_{3}\right)_{2} \mathrm{CHCH}_{2} \mathrm{C}\left(\mathrm{CH}_{3}\right) \mathrm{OH}$ & $\mathrm{NH}_{3}$ & 90 & 85 \\
\hline 7 & $\left(\mathrm{CH}_{3}\right)_{2} \mathrm{CCH}\left(\mathrm{CH}_{2}\right)_{2} \mathrm{C}\left(\mathrm{CH}_{3}\right) \mathrm{OH}$ & $\mathrm{NH}_{3}$ & 86 & 85 \\
\hline 8 & $\mathrm{PhOCH}_{2} \mathrm{CH}_{2} \mathrm{OH}$ & $\mathrm{PhCH}_{2} \mathrm{NH}_{2}$ & $*$ & 90 \\
\hline 9 & $\mathrm{PhOCH}_{2} \mathrm{CH}_{2} \mathrm{OH}$ & $c$-propylamine & $*$ & 92 \\
\hline 10 & $\mathrm{PhOCH}_{2} \mathrm{CH}_{2} \mathrm{OH}$ & $\left(\mathrm{CH}_{3}\right)_{2} \mathrm{CH}\left(\mathrm{CH}_{2}\right)_{2} \mathrm{NH}_{2}$ & $*$ & 92 \\
\hline 11 & $\mathrm{PhOCH}_{2} \mathrm{CH}_{2} \mathrm{OH}$ & $\operatorname{BocNH}\left(\mathrm{CH}_{2}\right)_{3} \mathrm{NH}_{2}$ & $*$ & 96 \\
\hline 12 & $\mathrm{PhOCH}_{2} \mathrm{CH}_{2} \mathrm{OH}$ & $2-\mathrm{MeOC}_{6} \mathrm{H}_{4} \mathrm{NH}_{2}$ & $*$ & 0 \\
\hline
\end{tabular}

* - Purity of compound was not determined. 
Another combinatorial synthesis of guanidines utilizes resin bound thiourea 70, which after conversion into the isothiourea $\mathbf{7 1}$ by treatment with iodomethane, reacted with primary or secondary amines to give polymer supported guanidines 72 . Cleavage provided access to a variety of guanidines $73^{33}$ (Scheme 26, Table 20).

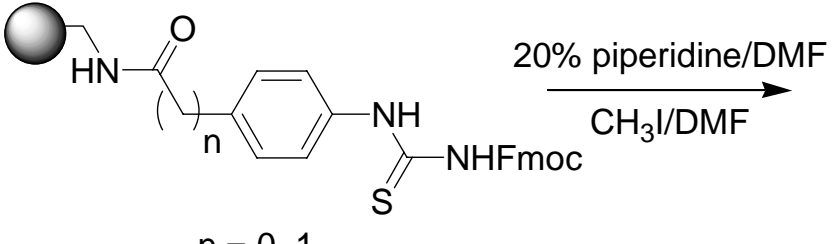

$\mathrm{n}=0,1 \quad 70$

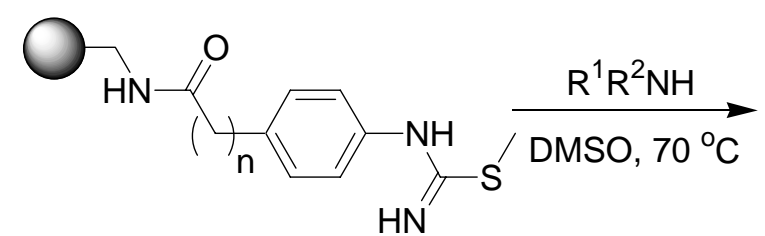

71

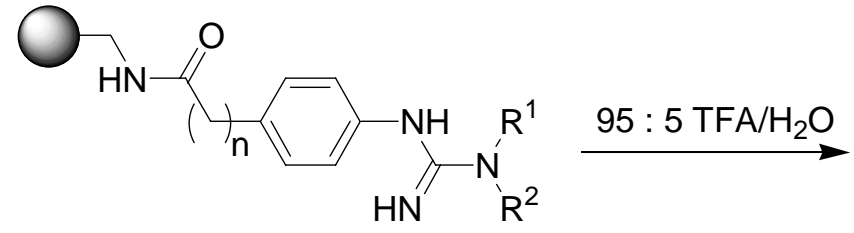

72

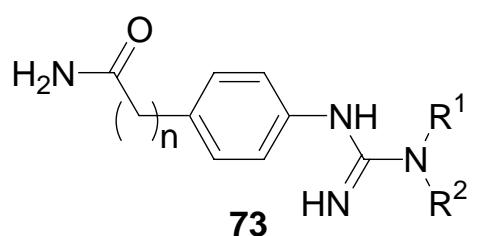

\section{Scheme 26}

Table 20. Synthesis of guanidines via resin-bound isothioureas (of Scheme 26)

\begin{tabular}{ccccc}
\hline Entry & $\mathrm{n}$ & $\mathrm{R}^{1} \mathrm{R}^{2} \mathrm{NH}$ & Purity, $\%$ & Yield, \% \\
\hline 1 & 0 & Morpholine & 73 & 77 \\
2 & 0 & Piperidine & 80 & 89 \\
3 & 0 & $N$-Methyl- $N$-phenethylamine & 82 & 73 \\
4 & 0 & 4-Methoxyphenethylamine & 40 & 64 \\
5 & 0 & $n$-Butylamine & 44 & 64 \\
6 & 1 & Morpholine & 88 & 95 \\
7 & 1 & Piperidine & 92 & 95 \\
8 & 1 & N-Methyl- $N$-phenethylamine & 89 & 93 \\
9 & 1 & 4-Methoxyphenethylamine & 79 & 72 \\
10 & 1 & $n$-Butylamine & 82 & 83 \\
\hline
\end{tabular}

Cyanoguanidines $\mathbf{7 6}$ were obtained in high yields by the reaction of substituted isothioureas 75 with cyanamide ${ }^{34}$ in boiling butanol in the presence of 1,4-diazabicyclo[2.2.2] octane (Scheme 27, Table 21).

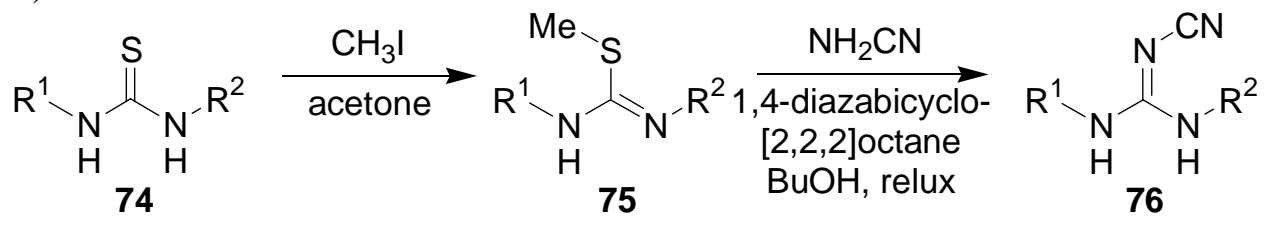

\section{Scheme 27}


Table 21. Synthesis of cyanoguanidines 76 in the presence of 1,4-diazabicyclo[2.2.2] octane (see Scheme 27)

\begin{tabular}{cccc}
\hline Entry & $\mathrm{R}^{1}$ & $\mathrm{R}^{2}$ & Yield, \% \\
\hline 1 & $\mathrm{Ph}$ & $\mathrm{Ph}$ & 68 \\
2 & $\mathrm{Ph}$ & $\mathrm{Me}$ & 70 \\
3 & $\mathrm{Ph}$ & Allyl & 77 \\
4 & $\mathrm{Ph}$ & $c$-Hexyl & 77 \\
5 & $c$-Hexyl & Me & 73 \\
6 & $c$-Hexyl & Allyl & 76 \\
7 & $c$-Hexyl & $c$-Hexyl & 73 \\
8 & $\mathrm{Bn}$ & Ph & 67 \\
9 & $\mathrm{Bn}$ & Me & 73 \\
10 & $\mathrm{Bn}$ & $c$-Hexyl & 69 \\
11 & $\mathrm{Bu}$ & $\mathrm{Ph}$ & 74 \\
12 & $\mathrm{Bu}$ & $c$-Hexyl & 70 \\
\hline
\end{tabular}

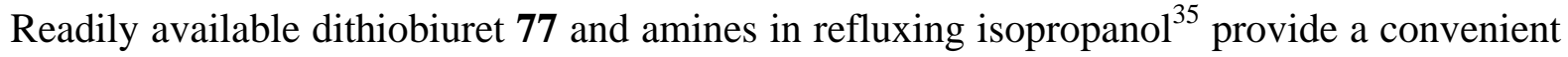
method for the preparation of mono $N$-substituted guanylthioureas $\mathbf{7 9}$ in moderate to very good yields (Scheme 28, Table 22).<smiles>CC(C)(C)C(C)(C)C(C)(C)C(N)=S</smiles>

77

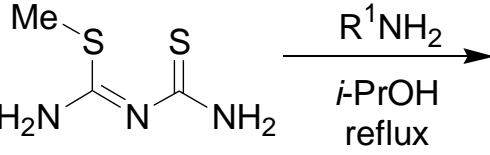

78<smiles>[R]N/C(N)=N/C(N)=S</smiles>

79

\section{Scheme 28}

Table 22. Synthesis of guanylthioutreas from dithiobiuret $\mathbf{7 8}$ by method of Scheme 28

\begin{tabular}{cccccc}
\hline Entry & $\mathrm{R}^{1}$ & Yield, \% & Entry & $\mathrm{R}^{1}$ & Yield, \% \\
\hline 1 & Ethyl & 34 & 6 & 2-(Acetylamino)ethyl & 56 \\
2 & C-Pr & 57 & 7 & 2-(t-Boc-amino)ethyl & 75 \\
3 & $n$-Pentyl & 68 & 8 & Benzyl & 75 \\
4 & n-Hexyl & 75 & 9 & 3-Pyridylmethyl & 58 \\
5 & 2-(Ethoxy)ethyl & 73 & 10 & Phenyl & 28 \\
\hline
\end{tabular}

\subsection{Carbodiimides and cyanamides}

$N$-Aryliminophosphoranes 80 were converted into $N^{1}, N^{2}, N^{3}$-triarylguanidines 83 in good yields by reaction with isocyanates $\mathbf{8 1}$ followed by treatment of the intermediate $N^{1}, N^{2}$ diarylcarbodiimides $\mathbf{8 2}$ with aromatic amines in the presence of TBAF. ${ }^{36}$ The presence of one equiv of TBAF is essential and the reaction takes place at room temperature within 10 min to 
give the desired guanidine (Scheme 29). As the commercially available THF solution of TBAF containes ca. $5 \mathrm{wt} \%$ water, it was pretreated with anhydrous $\mathrm{MgSO}_{4}$.

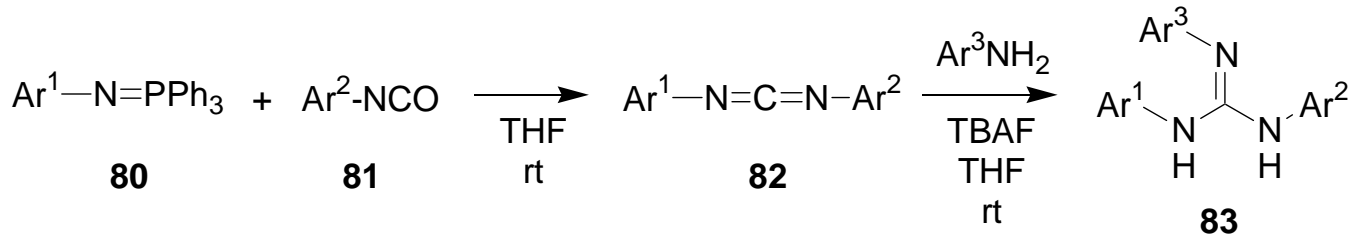

\section{Scheme 29}

Table 23. Synthesis of carbodiimides 82 and guanidines 83 (Scheme 29)

\begin{tabular}{ccccl}
\hline Entry & $\mathrm{Ar}^{1}$ & $\mathrm{Ar}^{2}$ & $\mathrm{Ar}^{3}$ & Yield, \% \\
\hline 1 & $2-\mathrm{BrC}_{6} \mathrm{H}_{4}$ & $4-\mathrm{CH}_{3} \mathrm{C}_{6} \mathrm{H}_{4}$ & $4-\mathrm{CH}_{3} \mathrm{C}_{6} \mathrm{H}_{4}$ & 73 \\
2 & $4-\mathrm{CH}_{3} \mathrm{C}_{6} \mathrm{H}_{4}$ & $2-\mathrm{BrC}_{6} \mathrm{H}_{4}$ & $4-\mathrm{CH}_{3} \mathrm{OC}_{6} \mathrm{H}_{4}$ & 80 \\
3 & $2-\mathrm{BrC}_{6} \mathrm{H}_{4}$ & $4-\mathrm{CH}_{3} \mathrm{C}_{6} \mathrm{H}_{4}$ & $4-\mathrm{ClC}_{6} \mathrm{H}_{4}$ & 75 \\
4 & $4-\mathrm{CH}_{3} \mathrm{C}_{6} \mathrm{H}_{4}$ & $2-\mathrm{BrC}_{6} \mathrm{H}_{4}$ & $4-\mathrm{NO}_{2} \mathrm{C}_{6} \mathrm{H}_{4}$ & 67 \\
5 & $\mathrm{C}_{6} \mathrm{H}_{5}$ & $2-\mathrm{BrC}_{6} \mathrm{H}_{4}$ & 4- $\mathrm{NO}_{2} \mathrm{C}_{6} \mathrm{H}_{4}$ & 52 \\
6 & 2-pyridyl & 4- $\mathrm{CH}_{3} \mathrm{C}_{6} \mathrm{H}_{4}$ & 2-pyridyl & 18 \\
\hline
\end{tabular}

Guanidinoacetic acids were prepared as outlined in Scheme 30 on solid support via treatment of anchored amines with intermediate solution-generated carbodiimides $\mathbf{8 5}^{37}$ This particular protocol is extremely practical. Conversion of thioureas 84 to carbodiimides 85 on treatment with Mukaiyama's reagent (2-chloro-1-methypyridinium iodide) is almost instantaneous at room temperature though brief sonication was desirable to accelerate solubilization of the reagent. The carbodiimides are very nonpolar and are generally isolated by extraction into hexanes and filtration through a short silica plug using the same solvent. Reaction times for addition of the carbodiimides 85 to Wang-supported glycine or alanine $\mathbf{8 6}$ varied, but the transformation was conveniently monitored via the ninhydrin test.

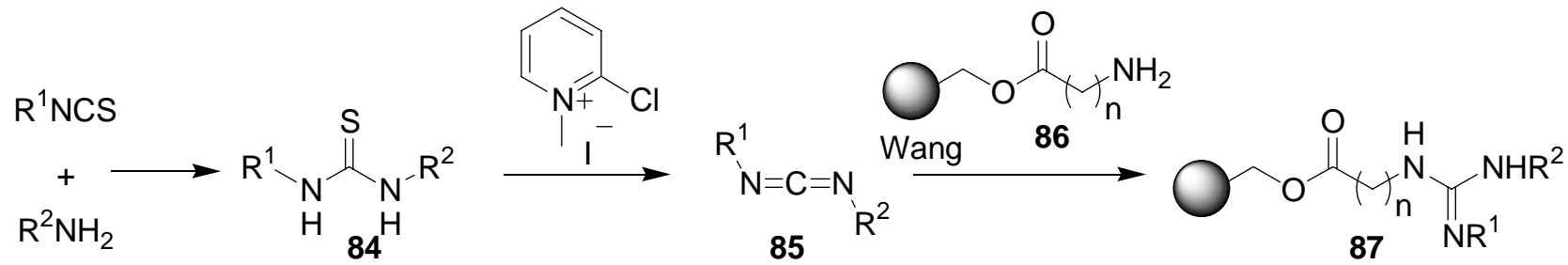

\section{Scheme 30}

Another approach via carbodiimides involves their preparation on solid support followed by the reaction with amines to furnish resin bound guanidines ${ }^{38}$ (Scheme 31). The sequence commenced with coupling of the $p$-bromomethyl benzoic acid to a primary amine of a Rinkextended macrocrown. The $p$-bromomethylbenzamide obtained underwent nucleophilic 
displacement with azide to afford the $\alpha$-azido-p-toluamide $\mathbf{8 8}$. Treatment with triphenylphosphine and phenyl isothiocyanate provided the carbodiimide $\mathbf{8 9}$, presumably via an in situ Staudinger reaction, to generate the intermediate iminophosphorane and subsequent azaWittig coupling with the isothiocyanate. Reaction of the carbodiimide with $N$-phenylpiperazine yielded a polymer-bound guanidine $\mathbf{9 0}$, which was cleaved with TFA/ $\mathrm{H}_{2} \mathrm{O}$ (95:5) to afford 91.

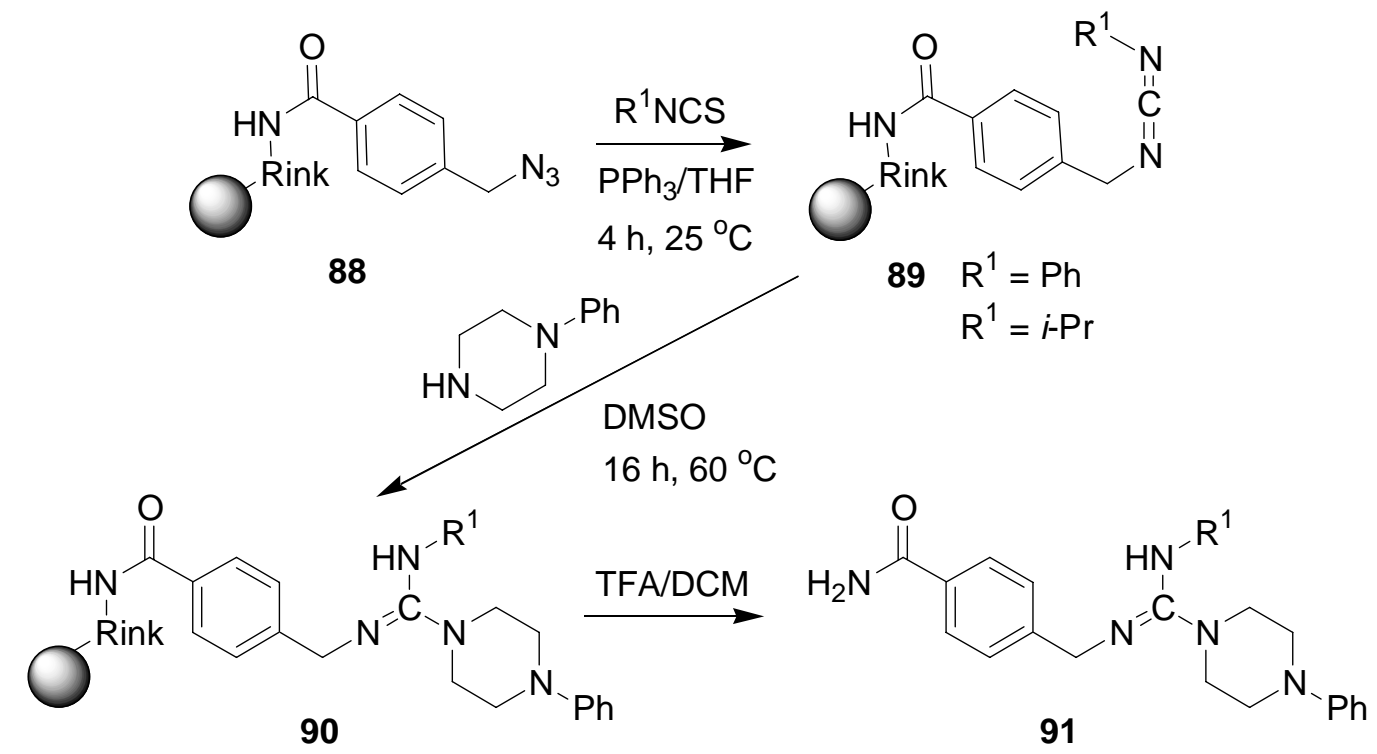

\section{Scheme 31}

Cyanamides serve as suitable starting materials for the preparation of guanidines: a recent example describes the formation of $\mathbf{9 2}$ in situ and immediate reaction with excess of amine ${ }^{39}$ to yield 93 (Scheme 32, Table 24).

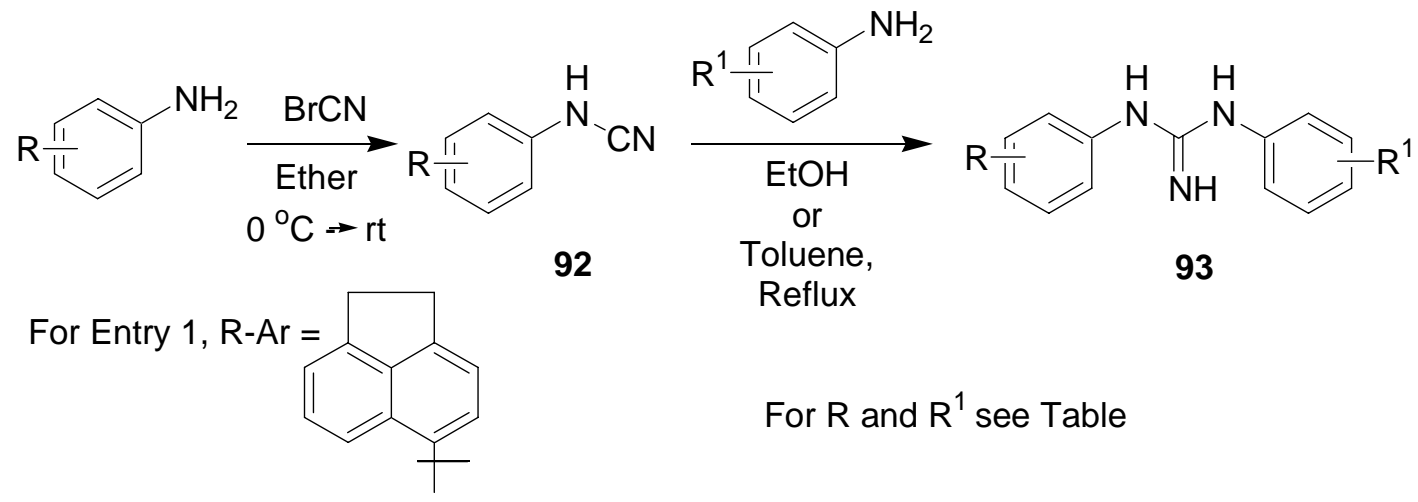

\section{Scheme 32}


Table 24. Synthesis of diarylguanidines 93 by method of Scheme 32

\begin{tabular}{cccccccc}
\hline Entry & $\mathrm{R}$ & $\mathrm{R}_{1}$ & Yield, \% & Entry & $\mathrm{R}$ & $\mathrm{R}_{1}$ & Yield, \% \\
\hline 1 & See Scheme & $\mathrm{s}-\mathrm{C}_{4} \mathrm{H}_{9}$ & 50 & 9 & $n-\mathrm{C}_{6} \mathrm{H}_{13}$ & $n-\mathrm{C}_{6} \mathrm{H}_{13}$ & 68 \\
2 & $\mathrm{CH}_{3}$ & $\mathrm{CH}_{3}$ & 20 & 10 & $\mathrm{C}_{6} \mathrm{H}_{5}$ & $\mathrm{C}_{6} \mathrm{H}_{5}$ & 8 \\
3 & $\mathrm{C}_{2} \mathrm{H}_{5}$ & $\mathrm{C}_{2} \mathrm{H}_{5}$ & 38 & 11 & $\mathrm{OC}_{6} \mathrm{H}_{5}$ & $\mathrm{OC}_{6} \mathrm{H}_{5}$ & 38 \\
4 & $i-\mathrm{C}_{3} \mathrm{H}_{7}$ & $\mathrm{i}-\mathrm{C}_{3} \mathrm{H}_{7}$ & 27 & 12 & $\mathrm{SC}_{6} \mathrm{H}_{5}$ & $\mathrm{SC}_{6} \mathrm{H}_{5}$ & 14 \\
5 & $s-\mathrm{C}_{4} \mathrm{H}_{9}$ & $\mathrm{~s}-\mathrm{C}_{4} \mathrm{H}_{9}$ & 32 & 13 & $\mathrm{OCH}_{2} \mathrm{C}_{6} \mathrm{H}_{5}$ & $t-\mathrm{C}_{4} \mathrm{H}_{9}$ & 53 \\
6 & $t-\mathrm{C}_{4} \mathrm{H}_{9}$ & $\mathrm{t}-\mathrm{C}_{4} \mathrm{H}_{9}$ & 40 & 14 & $\mathrm{OCH}_{2} \mathrm{C}_{6} \mathrm{H}_{5}$ & $n-\mathrm{C}_{4} \mathrm{H}_{9}$ & 44 \\
7 & $n-\mathrm{C}_{4} \mathrm{H}_{9}$ & $\mathrm{n}-\mathrm{C}_{4} \mathrm{H}_{9}$ & 20 & 15 & $n-\mathrm{OC}_{4} \mathrm{H}_{9}$ & $\left(\mathrm{CH}_{2}\right)_{4}-\mathrm{OH}$ & 67 \\
8 & $n-\mathrm{OC}_{4} \mathrm{H}_{9}$ & $\mathrm{n}-\mathrm{OC}_{4} \mathrm{H}_{9}$ & 27 & 16 & $\left(\mathrm{CH}_{2}\right)_{4}-\mathrm{OH}$ & $\left(\mathrm{CH}_{2}\right)_{4}-\mathrm{OH}$ & 79 \\
\hline
\end{tabular}

\subsection{Pyrazole-1-carboximidamides}

Pyrazole-1-carboximidamides $\mathbf{9 4}$ are now frequently used for the preparation of guanidines $\mathbf{9 5}$. The reaction of 2,4-dimethylpyrazole-1-carboximidamide with amines was considered in 1953 as abnormal because the initial desire was to substitute an $\mathrm{NH}_{2}$ group in analogy to ureas and related compounds $^{40 a}$ (Scheme 33). The guanidines prepared are listed in Table 25. For related work see references $40^{\mathrm{b}, \mathrm{c}}$.

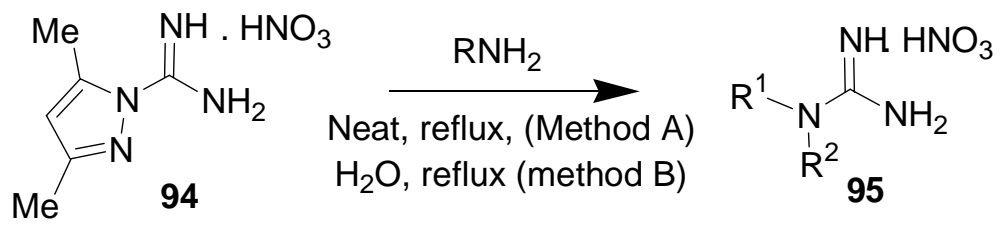

\section{Scheme 33}

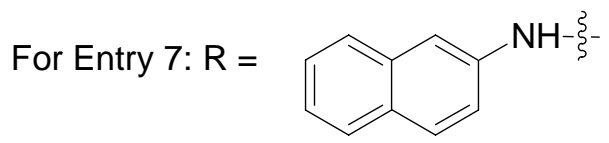

Table 25. Synthesis of guanidines from 2,4-dimethylpyrazole-1-carboximidamide nitrate (Scheme 33)

\begin{tabular}{ccccc}
\hline Entry & $\mathrm{R}^{1}$ & $\mathrm{R}^{2}$ & Method & Yield, \% \\
\hline 1 & $\mathrm{PhCONH}_{2}$ & $\mathrm{H}$ & B & 66 \\
2 & $\mathrm{PhCH}_{2}$ & $\mathrm{H}$ & B & 63 \\
3 & $-\left(\mathrm{CH}_{2}\right)_{2} \mathrm{O}\left(\mathrm{CH}_{2}\right)_{2}{ }^{-}$ & & A & 50 \\
4 & $\mathrm{PhCH} 2 \mathrm{CH} 2$ & $\mathrm{H}$ & $\mathrm{B}$ & 75 \\
5 & $-\left(\mathrm{CH}_{2}\right)_{5^{-}}$ & & B & 68 \\
6 & $-\left(\mathrm{CH}_{2}\right)_{4^{-}}$ & & A & 92 \\
7 & See Scheme & $\mathrm{H}$ & B & 67 \\
8 & 4- $\mathrm{CH}_{3} \mathrm{C}_{6} \mathrm{H}_{4} \mathrm{SO}_{2} \mathrm{NH}$ & $\mathrm{H}$ & B & 16 \\
\hline
\end{tabular}


A recent investigation showed that the presence of electron withdrawing groups in the pyrazole ring and/or on one or both of the carboximidamide nitrogens can be advantageous for the yield of guanidines and allow milder reaction conditions. Thus, di-Boc-4-nitropyrazole-1carboximidamide $\mathbf{9 6}$ has been used for the preparation of protected guanidines $97{ }^{41}$ (Scheme 34). A 4-nitro group in the pyrazole ring facilitates the reaction and gives higher yields (Table 26)

\section{Scheme 34}

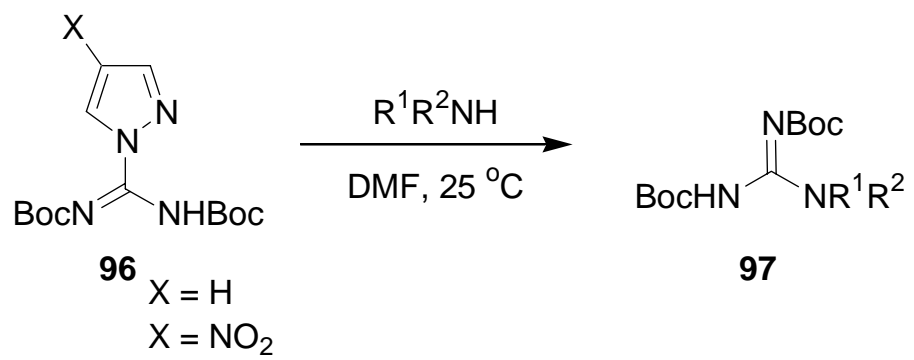

Table 26. Synthesis of guanidines from di-Boc-4-nitropyrazole-1- carboximidamide 97 (Scheme 34)

\begin{tabular}{cccc}
\hline Entry & $\mathrm{R}^{1} \mathrm{R}^{2} \mathrm{NH}$ & $\mathrm{X}=\mathrm{H}$, Yield, \% & $\mathrm{X}=\mathrm{NO}_{2}$, Yield, \% \\
\hline 1 & Benzylamine & 80 & 94 \\
2 & Piperidine & 70 & 82 \\
3 & Aniline & 11 & 78 \\
4 & Diisopropylamine & $<5$ & 64 \\
\hline
\end{tabular}

The use of $N$-Boc- $N$ '-tosylpyrazole-1-carboximidamide $\mathbf{1 0 0}$ is very efficient for the preparation of guanidines $\mathbf{1 0 1}$ and for coupling to peptides ${ }^{42}$ (Scheme 34). This reagent was also used for coupling with peptides in solution and on solid supports.

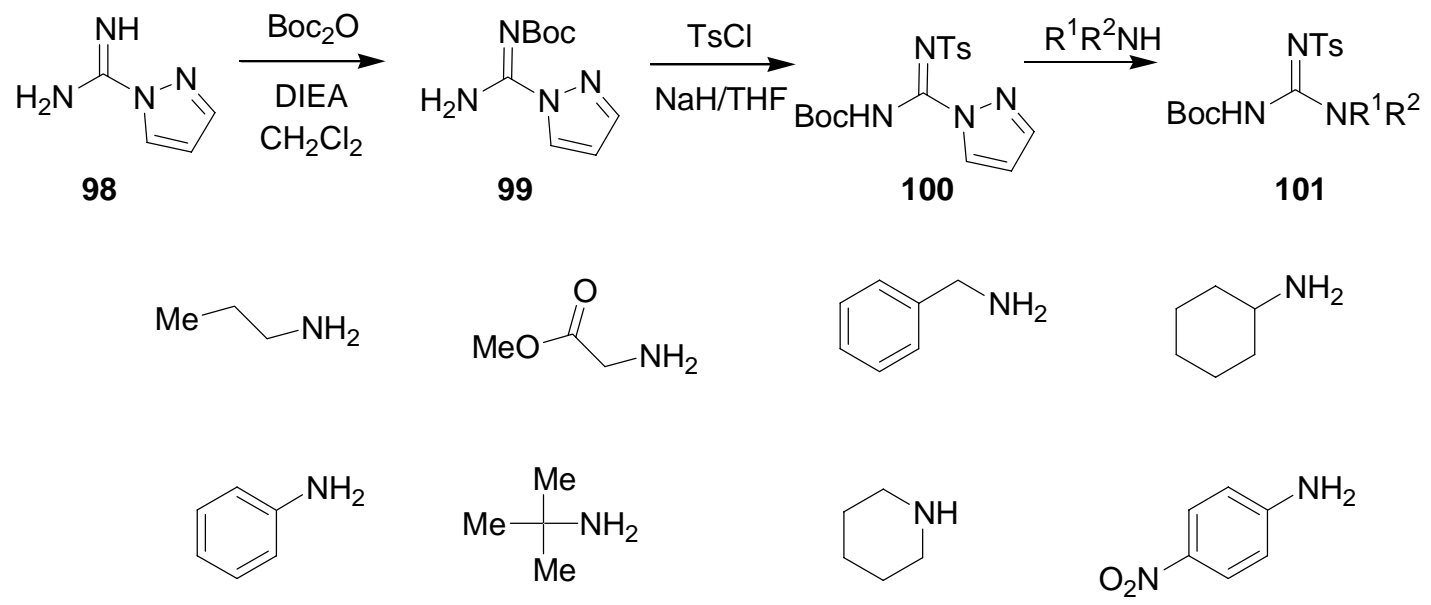

\section{Scheme 34}


The reactions of a variety of amines with di-Boc-pyrazole-1-carboximidamide $\mathbf{1 0 2}$ have also been examined $^{43}$ (Scheme 35, Table 27). In adidition to the guanylation of simple amines, several amino acids were converted into guanidino acids. Amino acids have a low solubility in many organic solvents, but reaction can be achieved in water, or aqueous acetonitrile to give satisfactory results.

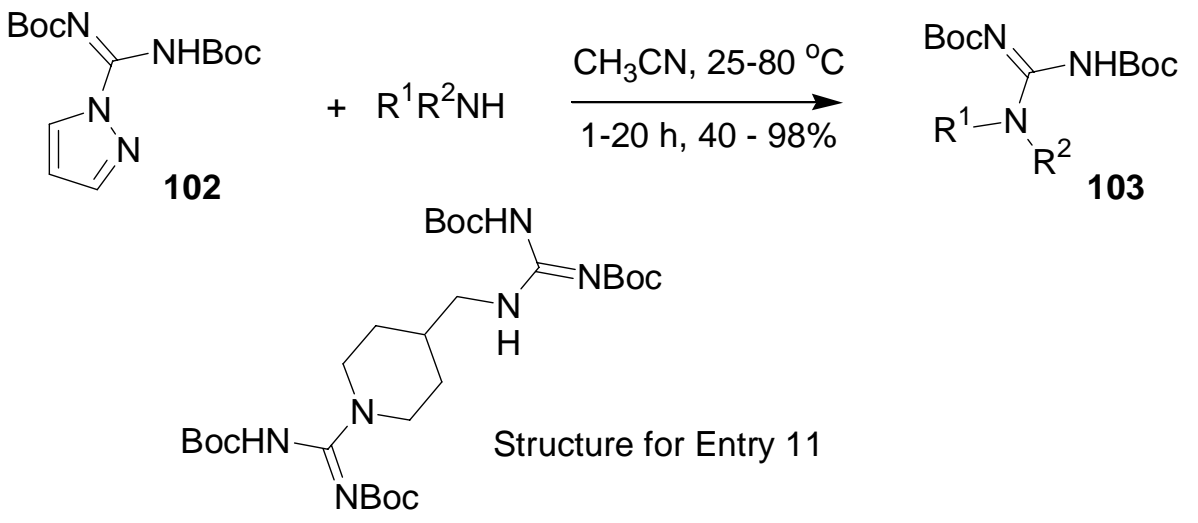

\section{Scheme 35}

Table 27. Synthesis of guanidines from di-Boc-pyrazole-1-carboximidamide (Scheme 35)

\begin{tabular}{|c|c|c|c|}
\hline Entry & $\mathrm{R}^{1}$ & $\mathrm{R}^{2}$ & Yield, \% \\
\hline 1 & $\mathrm{Bu}$ & $\mathrm{H}$ & 90 \\
\hline 2 & $t$-Bu & $\mathrm{H}$ & 93 \\
\hline 3 & $c$-Hex & $\mathrm{H}$ & 96 \\
\hline 4 & $\mathrm{Bn}$ & $\mathrm{H}$ & 91 \\
\hline 5 & Allyl & $\mathrm{H}$ & 98 \\
\hline 6 & $\mathrm{Ph}$ & $\mathrm{H}$ & 71 \\
\hline 7 & $4-\mathrm{MeOC}_{6} \mathrm{H}_{4}$ & $\mathrm{H}$ & 71 \\
\hline 8 & $4-\mathrm{NO}_{2} \mathrm{C}_{6} \mathrm{H}_{4}$ & $\mathrm{H}$ & 5 \\
\hline 9 & \multicolumn{2}{|c|}{$-\left(\mathrm{CH}_{2}\right)_{5^{-}}$} & 92 \\
\hline 10 & \multicolumn{2}{|c|}{$-\left(\mathrm{CH}_{2}\right)_{2} \mathrm{O}\left(\mathrm{CH}_{2}\right)_{2-}^{-}$} & 86 \\
\hline 11 & \multicolumn{2}{|c|}{$-(\mathrm{CH} 2)_{2} \mathrm{CH}\left(\mathrm{CH}_{2} \mathrm{NH}_{2}\right)\left(\mathrm{CH}_{2}\right)_{2}-$} & 67 \\
\hline 12 & Gly & $\mathrm{H}$ & 71 \\
\hline 13 & $\beta$-Ala & $\mathrm{H}$ & 38 \\
\hline 14 & 3-Aminobutyric acid & $\mathrm{H}$ & 70 \\
\hline 15 & 6-Aminocaproic acid & $\mathrm{H}$ & 76 \\
\hline 16 & \multicolumn{2}{|c|}{ Pro } & 73 \\
\hline 17 & Fmoc-Lys & $\mathrm{H}$ & 57 \\
\hline 18 & Cbz-Orn & $\mathrm{H}$ & 70 \\
\hline 19 & $\beta$-Ala-OMe & $\mathrm{H}$ & 89 \\
\hline
\end{tabular}


Pyrazole-1-carboximidamide connected to a solid support by an oxycarbonyl linker 104 can be acylated to $\mathbf{1 0 5}$ and thus becomes a potent reagent for the generation of guanidines 106 on solid supports $^{44}$ (Scheme 36) by utilizing electronically diverse acylating agents and amines. Guanidine derivatives from nitroanilines were obtained in good yields for a range of acylated derivatives of pyrazole-1-carboximidamide.

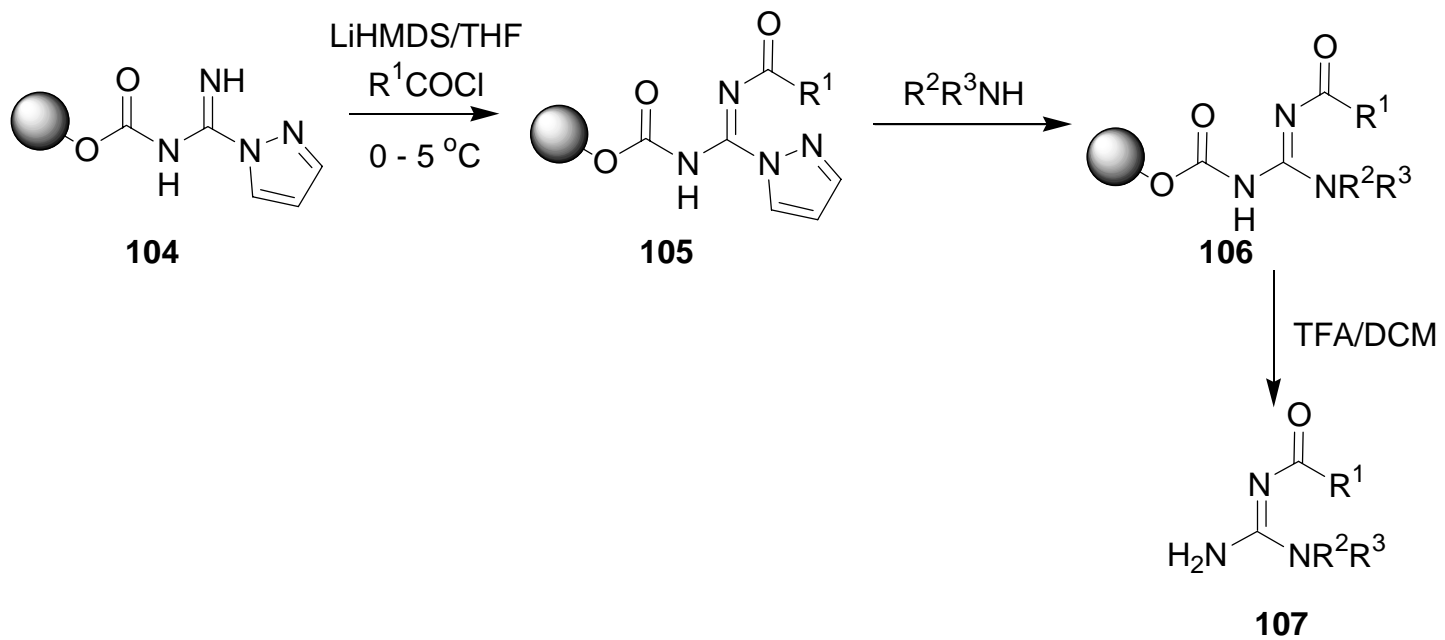

\section{Scheme 36}

\subsection{Triflyl guanidines}

Triflyl guanidines, first reported in 1998, are becoming popular reagents for the preparation of guanidines. $^{45}$ Di-Boc-triflylguanidine $\mathbf{1 1 0}$ and di -Cbz-triflylguanidine $\mathbf{1 1 3}$ are efficiently converted by various primary amines into guanidines $\mathbf{1 1 1}, \mathbf{1 1 4} .^{45-47}$ (Schemes 37, 38, Tables 28, 29).

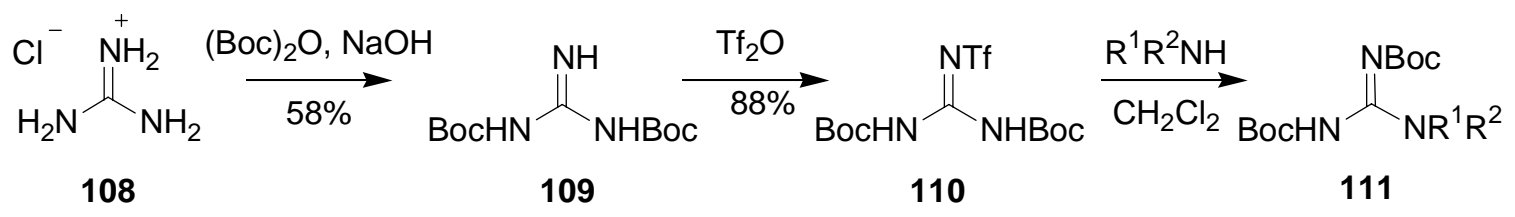

\section{Scheme 37}


Table 28. Synthesis of guanidines from di-Boc-triflylguanidine 110 (Scheme 37)

\begin{tabular}{|c|c|c|c|c|}
\hline Entry & $\mathrm{R}^{1} \mathrm{R}^{2} \mathrm{NH}$ & Conditions & Yield, \% & Ref. \\
\hline 1 & Benzylamine & $\mathrm{CH}_{2} \mathrm{Cl}_{2}, 0.5 \mathrm{~h}, \mathrm{rt}$ & 100 & 45 \\
\hline 2 & Cyclohexylamine & $\mathrm{CH}_{2} \mathrm{Cl}_{2}, 1.0 \mathrm{~h}, \mathrm{rt}$ & 99 & 45 \\
\hline 3 & $t$-Butylamine & $\mathrm{CH}_{2} \mathrm{Cl}_{2}, 8.0 \mathrm{~h}$, reflux & 75 & 45 \\
\hline 4 & 4-Pyrrolidine & $\mathrm{CH}_{2} \mathrm{Cl}_{2}, 2.0 \mathrm{~h}, \mathrm{rt}$ & 96 & 45 \\
\hline 5 & Aniline & $\mathrm{CH}_{2} \mathrm{Cl}_{2}, 24 \mathrm{~h}, \mathrm{rt}$ & 89 & 45 \\
\hline 6 & & $\mathrm{CH}_{2} \mathrm{Cl}_{2}, 4 \mathrm{~h}, \mathrm{rt}$ & 82 & 45 \\
\hline 7 & & $\mathrm{CH}_{2} \mathrm{Cl}_{2}, 4 \mathrm{~h}, \mathrm{rt}$ & 85 & 45 \\
\hline 8 & & $\mathrm{CH}_{2} \mathrm{Cl}_{2}, 4 \mathrm{~h}, \mathrm{rt}$ & 88 & 45 \\
\hline 9 & $\mathrm{CH}_{3}\left(\mathrm{CH}_{2}\right)_{4} \mathrm{NH}_{2}$ & $\mathrm{CH}_{2} \mathrm{Cl}_{2}, 1 \mathrm{~h}, \mathrm{rt}$ & 98 & 45 \\
\hline 10 & Piperidine & $\mathrm{CHCl}_{3}, 20 \mathrm{~h}, \mathrm{rt}$ & 85 & 45 \\
\hline 11 & Morpholine & - & 86 & 45 \\
\hline 12 & Piperazine & - & 100 & 45 \\
\hline 13 & Monoethanolamine & $\mathrm{CH}_{2} \mathrm{Cl}_{2}, 2 \mathrm{~h}, \mathrm{rt}$ & 100 & 45 \\
\hline 14 & & $\begin{array}{c}\mathrm{H}_{2} \mathrm{O} \text {, Dioxane } \\
\mathrm{CH}_{3} \mathrm{CN}\end{array}$ & $22-49$ & 46 \\
\hline 15 & & $\mathrm{CH}_{2} \mathrm{Cl}_{2}$ & 42 & 47 \\
\hline 16 & & $\mathrm{CH}_{2} \mathrm{Cl}_{2}$ & 21 & 47 \\
\hline 17 & & $\mathrm{CH}_{2} \mathrm{Cl}_{2}$ & 38 & 47 \\
\hline 18 & & $\mathrm{CH}_{2} \mathrm{Cl}_{2}$ & 39 & 47 \\
\hline 19 & & $\mathrm{CH}_{2} \mathrm{Cl}_{2}$ & 42 & 47 \\
\hline
\end{tabular}




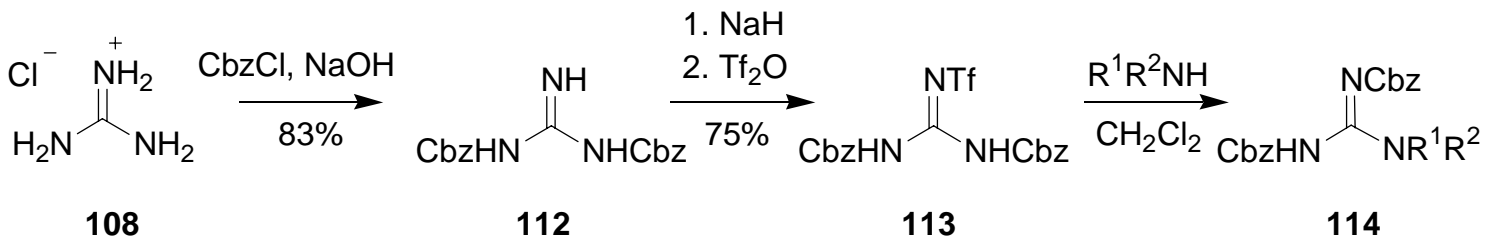

\section{Scheme 38}

Table 29. Synthesis of guanidines from di-Cbz-triflylguanidine 113 (Scheme 38)

\begin{tabular}{llll}
\hline Entry & $\mathrm{R}^{1} \mathrm{R}^{2} \mathrm{NH}$ & Conditions & Yield, \% \\
\hline 1 & Benzylamine & $\mathrm{CH}_{2} \mathrm{Cl}_{2}, 1.0 \mathrm{~h}, \mathrm{rt}$ & 94 \\
2 & Aniline & $\mathrm{CH}_{2} \mathrm{Cl}_{2}, 1 \mathrm{~h}, \mathrm{rt}$ & 98 \\
3 & FmocHN_COOH & $\mathrm{CH}_{2} \mathrm{Cl}_{2}, 4 \mathrm{~h}, \mathrm{rt}$ & 85 \\
& & & \\
& & & \\
& & & \\
& $\left(\mathrm{CH}_{2}\right)_{3}$ & & \\
$\mathrm{NH}_{2}$ & &
\end{tabular}

Primary and secondary amines were also converted into guanidines 116 by triflylguanidines $\mathbf{1 1 5}^{48}$ (Scheme 39, Table 30).

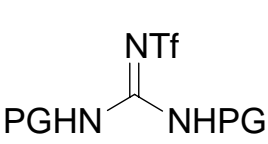

115

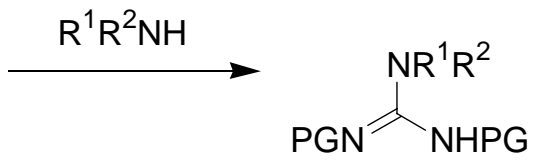

116

Scheme 39

Table 30. Conversion of secondary amines into guanidines by di-protected triflylguanidine 115 (Scheme 39)

\begin{tabular}{|c|c|c|c|c|}
\hline Entry & PG & $\mathrm{R}^{1}$ & $\mathrm{R}^{2}$ & Yield, \% \\
\hline 1 & Boc & & $\mathrm{H}$ & 100 \\
\hline 2 & Boc & & $\mathrm{H}$ & 88 \\
\hline 3 & Boc & & & 49 \\
\hline 4 & $\mathrm{Cbz}$ & & $\mathrm{H}$ & 95 \\
\hline
\end{tabular}

\subsection{Aminoiminomethane-sulfonic and -sulfinic acids}

Aminoiminosulfonic and -sulfinic acids, prepared by the oxidation of thioureas, are useful reagents for the synthesis of guanidines. 
Marianoff and coworkers described a practical two-step procedure based on thioureas 117 oxidized with $\mathrm{H}_{2} \mathrm{O}_{2}{ }^{49}$ A high yield of pure sulfonic acid $\mathbf{1 1 8}$ was obtained rapidly in a short reaction time when the reaction was run as a slurry in water. The rate of reaction was dependent on the concentration of catalyst $\left(\mathrm{Na}_{2} \mathrm{MoO}_{4}\right)$ employed. In general, the sulfonic acid derivatives are stable at room temperature and are the preferred intermediates. The oxidation products were isolated by filtration and air-dried for use in the displacement reaction (Scheme 40, Table 31).

The second step of the sequence, displacement of the oxidized sulfur with amine nucleophiles, to give 119 was carried out under mild conditions (Scheme 40). Yields of the displacement reactions are reported in Table 32.

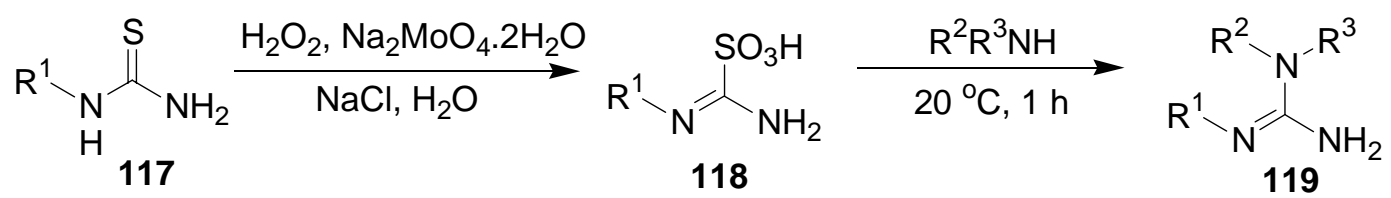

\section{Scheme 40}

Table 31. Oxidation of thioureas $\mathbf{1 1 7}$ to sulfonic acid $\mathbf{1 1 8}$

\begin{tabular}{lll}
\hline Entry & $\mathrm{R}^{1}$ & Yield, \% \\
\hline 1 & $\mathrm{Ph}$ & 86 \\
2 & $n-\mathrm{Pr}$ & 56 \\
3 & $2-\mathrm{CH}_{3} \mathrm{C}_{6} \mathrm{H}_{4}$ & 83 \\
4 & $4-\mathrm{FC}_{6} \mathrm{H}_{4}$ & 76 \\
\hline
\end{tabular}

Table 32. Synthesis of guanidines 119 from thiourea derived sulfonic acid 118.

\begin{tabular}{|c|c|c|c|c|}
\hline Entry & $\mathrm{R}^{1}$ & $\mathrm{R}^{2}$ & $\mathrm{R}^{3}$ & Yield, \% \\
\hline 1 & $\mathrm{Ph}$ & $t$-Butyl & $\mathrm{H}$ & 99 \\
\hline 2 & $\mathrm{Ph}$ & i-Butyl & $\mathrm{H}$ & 56 \\
\hline 3 & $\mathrm{Ph}$ & sec-Butyl & $\mathrm{H}$ & 50 \\
\hline 4 & $\mathrm{Ph}$ & $\mathrm{Ph}$ & $\mathrm{H}$ & 99 \\
\hline 5 & $\mathrm{Ph}$ & $4-\mathrm{CH}_{3} \mathrm{OC}_{6} \mathrm{H}_{4}$ & $\mathrm{H}$ & 77 \\
\hline 6 & $n-\operatorname{Pr}$ & sec-Butyl & $\mathrm{H}$ & 62 \\
\hline 7 & $\mathrm{Ph}$ & c-Hexyl & $\mathrm{H}$ & 72 \\
\hline 8 & $\mathrm{Ph}$ & $4-\mathrm{ClC}_{6} \mathrm{H}_{4}$ & $\mathrm{H}$ & 50 \\
\hline 9 & $\mathrm{Ph}$ & $2-\mathrm{CH}_{3}-4-\mathrm{CH}_{3} \mathrm{OC}_{6} \mathrm{H}_{4}$ & $\mathrm{H}$ & 51 \\
\hline 10 & $\mathrm{Ph}$ & $4-\mathrm{NO}_{2} \mathrm{C}_{6} \mathrm{H}_{4}$ & $\mathrm{H}$ & 84 \\
\hline 11 & $n-\operatorname{Pr}$ & i-Butyl & $\mathrm{H}$ & 23 \\
\hline 12 & $n-\operatorname{Pr}$ & n-Butyl & $\mathrm{H}$ & 60 \\
\hline 13 & $\mathrm{Ph}$ & \multicolumn{2}{|c|}{$-\left(\mathrm{CH}_{2}\right)_{4^{-}}$} & 73 \\
\hline 14 & $\mathrm{Ph}$ & \multicolumn{2}{|c|}{$-\left(\mathrm{CH}_{2}\right)_{2} \mathrm{O}\left(\mathrm{CH}_{2}\right)_{2^{-}}$} & 79 \\
\hline
\end{tabular}


Similar investigation on the use of aminoiminomethansulfonic acids $\mathbf{1 2 1}$ has been described for the conversion of amino acids into a guanidino acids $122{ }^{50}$ (Scheme 41, Table 33). This method is advantageous for the preparation of di- and tri-substituted guanidine acids due to their synthesis from isothiouronium compounds.

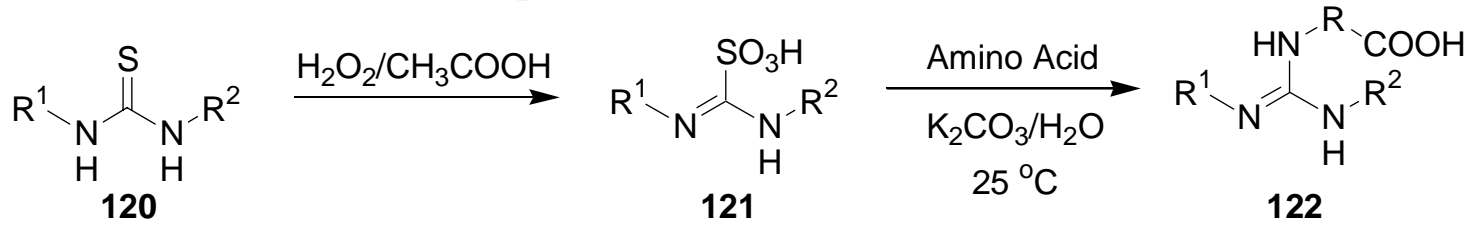

\section{Scheme 41}

Table 33. Synthesis of guanidines 122 via oxidized thioureas 121

\begin{tabular}{lllll}
\hline Entry & Amino Acid & $\mathrm{R}^{1}$ & $\mathrm{R}^{2}$ & Yield, \% \\
\hline 1 & 3-Aminopropanoic Acid & $\mathrm{H}$ & $\mathrm{H}$ & 75 \\
2 & DL-Alanine & $\mathrm{H}$ & $\mathrm{H}$ & 5 \\
3 & p-Aminobenzoic Acid & $\mathrm{H}$ & $\mathrm{H}$ & 80 \\
4 & 4-Aminobutanoic Acid & $\mathrm{H}$ & $\mathrm{H}$ & 50 \\
5 & 5-Aminopentanoic Acid & $\mathrm{H}$ & $\mathrm{H}$ & 55 \\
6 & Glycine & $\mathrm{H}$ & $\mathrm{H}$ & 80 \\
7 & L-Iisoleucine & $\mathrm{H}$ & $\mathrm{H}$ & 5 \\
8 & L-Leucine & $\mathrm{H}$ & $\mathrm{H}$ & $-{ }^{\mathrm{a}}$ \\
9 & DL-Methionine & $\mathrm{H}$ & $\mathrm{H}$ & 60 \\
10 & L-Phenylalanine & $\mathrm{H}$ & $\mathrm{H}$ & 45 \\
11 & L-Proline & $\mathrm{H}$ & $\mathrm{H}$ & $-{ }^{\mathrm{a}}$ \\
12 & DL-Serine & $\mathrm{H}$ & $\mathrm{H}$ & 50 \\
13 & DL-Valine & $\mathrm{H}$ & $\mathrm{H}$ & 55 \\
14 & 3-Aminopropanoic Acid & $\mathrm{H}$ & $\mathrm{Ph}$ & 70 \\
15 & p-Aminobenzoic Acid & $\mathrm{H}$ & $\mathrm{Ph}$ & 65 \\
16 & 4-Aminobutanoic Acid & $\mathrm{H}$ & $\mathrm{Ph}$ & 0 \\
17 & Glycine & $\mathrm{H}$ & $\mathrm{Ph}$ & 85 \\
18 & L-Leucine & $\mathrm{H}$ & $\mathrm{Ph}$ & 0 \\
19 & 3-Aminopropanoic Acid & $\mathrm{Ph}$ & $\mathrm{Ph}$ & 25 \\
20 & p-Aminobenzoic Acid & $\mathrm{Ph}$ & $\mathrm{Ph}$ & 25 \\
21 & Glycine & $\mathrm{Ph}$ & $\mathrm{Ph}$ & 35 \\
\hline
\end{tabular}

Continued optimization of the protocol for guanidine preparation led to use of the oxidized product without isolation $^{51}$ (Scheme 42). Oxidizing agents are compared in Table 32 and the oxidation rate for aliphatic derivatives was improved by addition of a catalytic amount of triethyl amine. The guanidines $\mathbf{1 2 4}$ were obtained by treatment with amines in water with short reaction times, excellent conversion, and simple isolation. However, trisubstituted thioureas resisted the oxidation. 


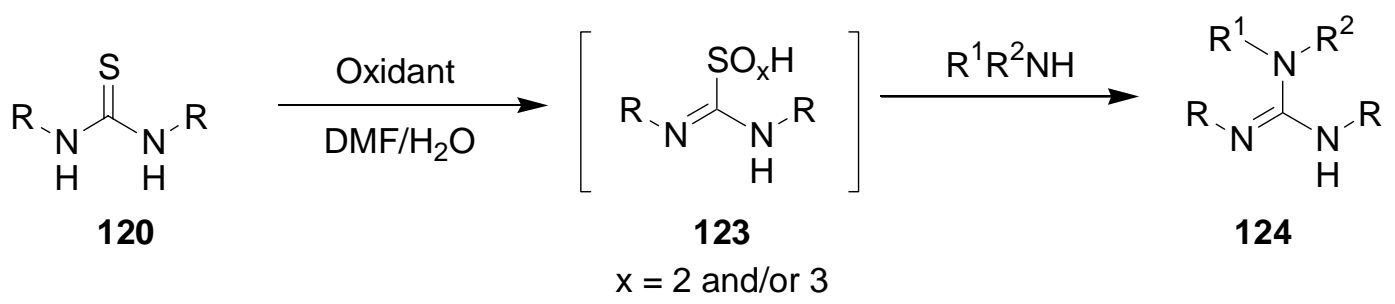

\section{Scheme 42}

Table 34. Synthesis of guanidines 124 via nonisolatable intermediate oxidized thioureas 123

\begin{tabular}{cccccc}
\hline Entry & $\mathrm{R}$ & $\mathrm{R}^{1}$ & $\mathrm{R}^{2}$ & $\begin{array}{c}\mathrm{NaIO}_{4} \\
\text { Yield, \% }\end{array}$ & $\begin{array}{c}\mathrm{NaClO}_{2} \\
\text { Yield, \% }\end{array}$ \\
\hline 1 & $\mathrm{Ph}$ & $\mathrm{H}$ & $\mathrm{H}$ & 76 & 80 \\
2 & $0^{-}$-Tolyl & $\mathrm{H}$ & $\mathrm{H}$ & 80 & 76 \\
3 & $\mathrm{C}_{6} \mathrm{H}_{11}$ & $\mathrm{H}$ & $\mathrm{H}$ & 72 & 67 \\
4 & $\mathrm{Ph}$ & $-\left(\mathrm{CH}_{2}\right)_{2} \mathrm{O}\left(\mathrm{CH}_{2}\right)_{2}{ }^{-}$ & 75 & 72 \\
5 & $\mathrm{Ph}$ & $\mathrm{H}$ & $\mathrm{C}_{6} \mathrm{H}_{11}$ & 84 & 81 \\
6 & $\mathrm{Ph}$ & $\mathrm{H}$ & $\mathrm{Benzyl}$ & 68 & 65 \\
7 & $\mathrm{Ph}$ & $\mathrm{Et}$ & $\mathrm{Et}$ & 76 & 80 \\
8 & $\mathrm{Ph}$ & $\mathrm{H}$ & $\mathrm{Et}$ & 75 & 72 \\
9 & $\mathrm{Ph}$ & $\mathrm{C}_{6} \mathrm{H}_{11}$ & $\mathrm{C}_{6} \mathrm{H}_{11}$ & 60 & 55 \\
\hline
\end{tabular}

In a recent, one-pot procedure, quaternary ammonium permanganate in the presence of amine showed advantages over other oxidizing agents for thioureas $\mathbf{1 2 0}^{52}$ (Scheme 43, Table 33).

Table 35. Synthesis of guanidines from oxidation of thioureas by quaternary ammonium permanganate (Scheme 43)

\begin{tabular}{lllll}
\hline Entry & $\mathrm{R}^{1}$ & $\mathrm{R}^{2}$ & $\mathrm{R}^{3}$ & Yield, \% \\
\hline 1 & $\mathrm{Ph}$ & $c-\mathrm{C}_{6} \mathrm{H}_{11}$ & $\mathrm{H}$ & 92 \\
2 & $\mathrm{Ph}$ & Ethyl & Ethyl & 95 \\
3 & $\mathrm{Ph}$ & $\mathrm{H}$ & $\mathrm{H}$ & 90 \\
4 & $\mathrm{Ph}$ & Benzyl & $\mathrm{H}$ & 87 \\
5 & $\mathrm{Ph}$ & $n-\mathrm{Bu}$ & $\mathrm{H}$ & 86 \\
6 & $o-\mathrm{Tolyl}$ & $\mathrm{H}$ & $\mathrm{H}$ & 82 \\
7 & $\mathrm{Ph}$ & $c-\mathrm{C}_{6} \mathrm{H}_{11}$ & $c-\mathrm{C}_{6} \mathrm{H}_{11}$ & 82 \\
8 & $\mathrm{Ph}$ & $i-\mathrm{Propyl}_{1}-\mathrm{Propyl}_{2}$ & 78 \\
9 & $\mathrm{Ph}$ & $-\left(\mathrm{CH}_{2}\right)_{2} \mathrm{O}\left(\mathrm{CH}_{2}\right)_{2^{-}}$ & 89 \\
10 & $2,6-$ Diethylphenyl & $-\left(\mathrm{CH}_{2}\right)_{2} \mathrm{O}\left(\mathrm{CH}_{2}\right)_{2^{-}}$ & 95 \\
\hline
\end{tabular}




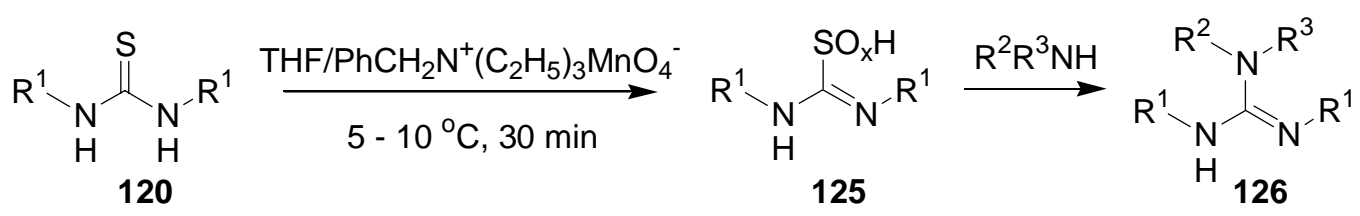

\section{Scheme 43}

\subsection{Benzotriazole and imidazole-activated reagents}

Benzotriazole-1-carboxamidinium tosylate $\mathbf{1 2 9}^{53}$ prepared from benzotriazole $\mathbf{1 2 8}$ with cyanamide 127 in refluxing 1,4-dioxane in the presence of $p$ - $\mathrm{TsOH}$, is an efficient general reagent for the synthesis of mono and disubstituted guanidines 130. Reactions are conveniently carried out using equimolar amine in (i) DMF-diisopropylethylamine at room temperature, (ii) acetonitrile or (iii) the absence of solvent. Product isolation is facile as the precipitated guanidine can be filtered from the ether soluble benzotriazole by-poduct when DMF is used. The product precipitates during the reaction, while in the absence of solvent product can be isolated chromatographically. Under mild conditions, benzotriazole-1-carboxamidinium tosylate gives guanidines in moderate to good yields and offers advantages over previous procedures (Scheme 44, Table 36).

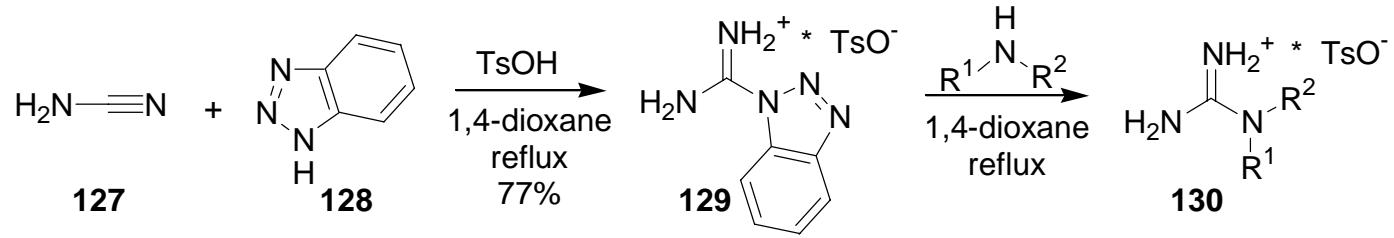

\section{Scheme 44}

Table 36. Synthesis of guanidines from benzotriazole-1-carboxamidinium tosylate

\begin{tabular}{|c|c|c|c|}
\hline Entry & Amine & Guanidine & Yield, \% \\
\hline 1 & $\mathrm{Me}_{2} \mathrm{NH}$ & & 69 \\
\hline 2 & & & 84 \\
\hline 3 & & & 68 \\
\hline 4 & $\mathrm{C}_{4} \mathrm{H}_{9} \mathrm{NH}_{2}$ & Ts & 55 \\
\hline 5 & & & 68 \\
\hline 6 & & & 86 \\
\hline 7 & & & 71 \\
\hline 8 & $\mathrm{C}_{6} \mathrm{H}_{13} \mathrm{NH}_{2}$ & T & 67 \\
\hline & & $\mathrm{NH}_{2}$ & \\
\hline
\end{tabular}


Modification of benzotriazole-1-carboxamidine by introducing electron withdrawing groups, Boc on both nitrogens of the amidine moiety and nitro or chloro group in benzotriazole to give 131, enhanced the ability of the benzotriazole moiety as a leaving group ${ }^{54}$ (Scheme 45 ).

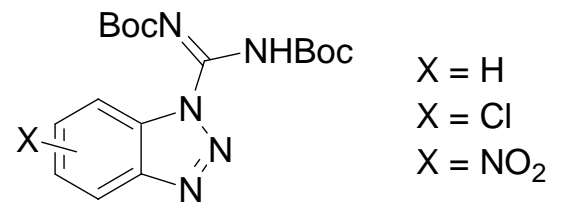

\section{Scheme 45}

131

Di(benzotriazolyl)carboximidamide $\mathbf{1 3 2}$ has been developed as a new guanylating agent, for the synthesis of tri- $\mathbf{1 3 6}$ and tetra-substituted guanidines $135 .{ }^{55}$ The sequential condensation of two amines with di(benzotriazolyl)carboximidamide is insensitive to electronic and steric effects allowing the use of a wide variety of amines and guanidines as free bases. The products were obtained in high yields under neutral and mild conditions using an easy purification protocol (Scheme 46, Tables 37, 38).

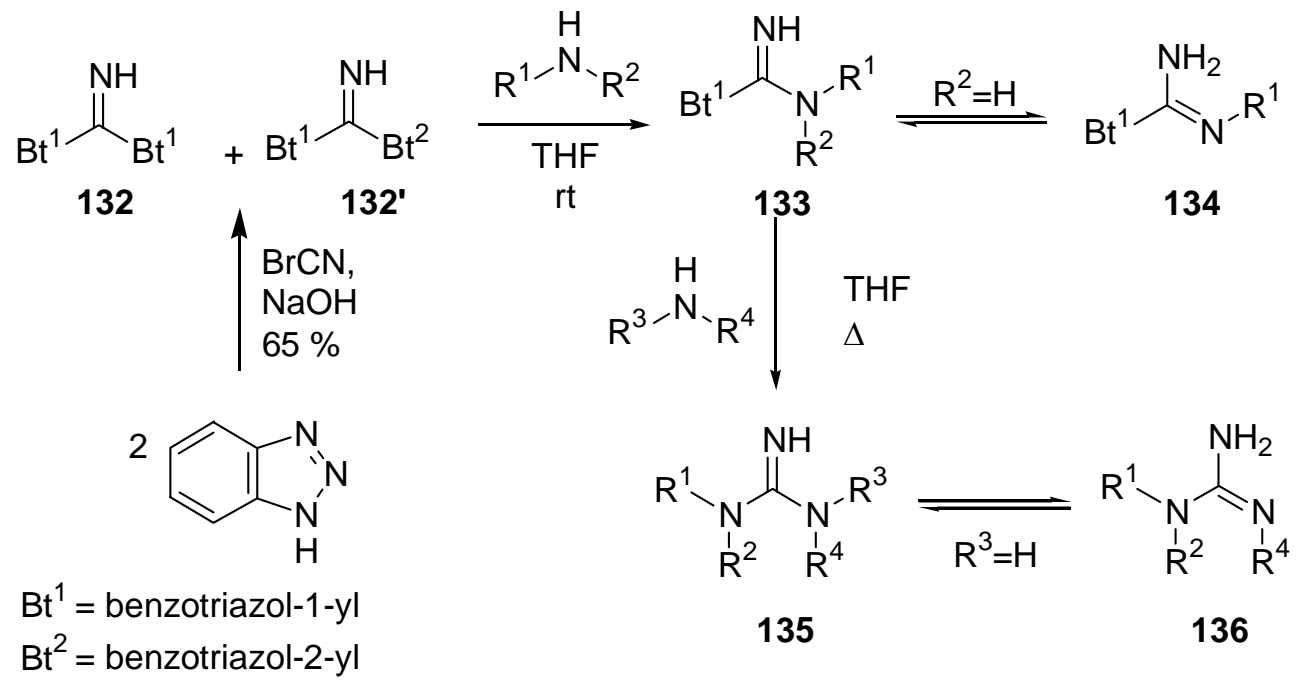

\section{Scheme 46}

Table 37. Preparation of benzotriazole-1-carboximidamides 133 (of Scheme 46)

\begin{tabular}{cccc}
\hline Entry & $\mathrm{R}^{1}$ & $\mathrm{R}^{2}$ & Yield, (\%) \\
\hline 1 & $\mathrm{H}$ & $\mathrm{C}_{6} \mathrm{H}_{5}$ & 80 \\
2 & $\mathrm{H}$ & $n-\mathrm{C}_{5} \mathrm{H}_{11}$ & 74 \\
3 & $\mathrm{H}$ & $\mathrm{CH}_{2} \mathrm{C}_{6} \mathrm{H}_{5}$ & 68 \\
4 & \multicolumn{2}{c}{$-\left(\mathrm{CH}_{2}\right)_{4}{ }^{-}$} & 71 \\
5 & \multicolumn{2}{c}{$-\left(\mathrm{CH}_{2}\right)_{2} \mathrm{O}\left(\mathrm{CH}_{2}\right)_{2}{ }^{-}$} & 68 \\
6 & $\mathrm{CH}\left(\mathrm{CH}_{3}\right)_{2}$ & $\mathrm{CH}\left(\mathrm{CH}_{3}\right)_{2}$ & 68 \\
\hline
\end{tabular}


Table 38. Preparation of guanidines 135 (of Scheme 46)

\begin{tabular}{|c|c|c|c|c|c|}
\hline Entry & $\mathrm{R}^{2}$ & $\mathrm{R}^{3}$ & $\mathrm{R}^{4}$ & $\begin{array}{l}\text { Reaction time } \\
\text { (h) }\end{array}$ & $\begin{array}{l}\text { Yield } \\
(\%)\end{array}$ \\
\hline 1 & $-\left(\mathrm{CH}_{2}\right)_{2} \mathrm{O}\left(\mathrm{CH}_{2}\right)_{2-}$ & $\mathrm{C}_{6} \mathrm{H}_{5}$ & $\mathrm{H}$ & 10 & 64 \\
\hline 2 & $-\left(\mathrm{CH}_{2}\right)_{2} \mathrm{O}\left(\mathrm{CH}_{2}\right)_{2^{-}}$ & 4- $\mathrm{CH}_{3} \mathrm{C}_{6} \mathrm{H}_{4}$ & $\mathrm{H}$ & 12 & 74 \\
\hline 3 & $-\left(\mathrm{CH}_{2}\right)_{2} \mathrm{O}\left(\mathrm{CH}_{2}\right)_{2^{-}}$ & $\mathrm{C}_{6} \mathrm{H}_{5} \mathrm{CH}_{2}$ & $\mathrm{H}$ & 12 & 71 \\
\hline 4 & $-\left(\mathrm{CH}_{2}\right)_{2} \mathrm{O}\left(\mathrm{CH}_{2}\right)_{2}-$ & $\mathrm{C}_{6} \mathrm{H}_{5}$ & $\mathrm{CH}_{3}$ & 18 & 85 \\
\hline 5 & $-\left(\mathrm{CH}_{2}\right)_{4^{-}}$ & $\mathrm{C}_{6} \mathrm{H}_{5}$ & $\mathrm{H}$ & 12 & 68 \\
\hline 6 & $-\left(\mathrm{CH}_{2}\right)_{4^{-}}$ & $4-\mathrm{CH}_{3} \mathrm{OC}_{6} \mathrm{H}_{4}$ & $\mathrm{H}$ & 17 & 60 \\
\hline 7 & $\mathrm{CH}\left(\mathrm{CH}_{3}\right)_{2} \quad \mathrm{CH}\left(\mathrm{CH}_{3}\right)_{2}$ & $4-\mathrm{CH}_{3} \mathrm{OC}_{6} \mathrm{H}_{4}$ & $\mathrm{H}$ & 15 & 48 \\
\hline
\end{tabular}

Analogous to the di(benzotriazolyl)carboximidamide reagent 132, di(imidazol-1yl)carboximidamide 138 was later synthesized by treatment of cyanogens bromide with imidazole. ${ }^{56}$ Reagent 138 can also be converted into substituted guanidines $\mathbf{1 4 1}$ by sequential displacement of imidazole moieties with amines. Further, through its $N$-cyano derivative 139 it provides an access to substituted cyanoguanidines 143 (Scheme 47, Table 38).

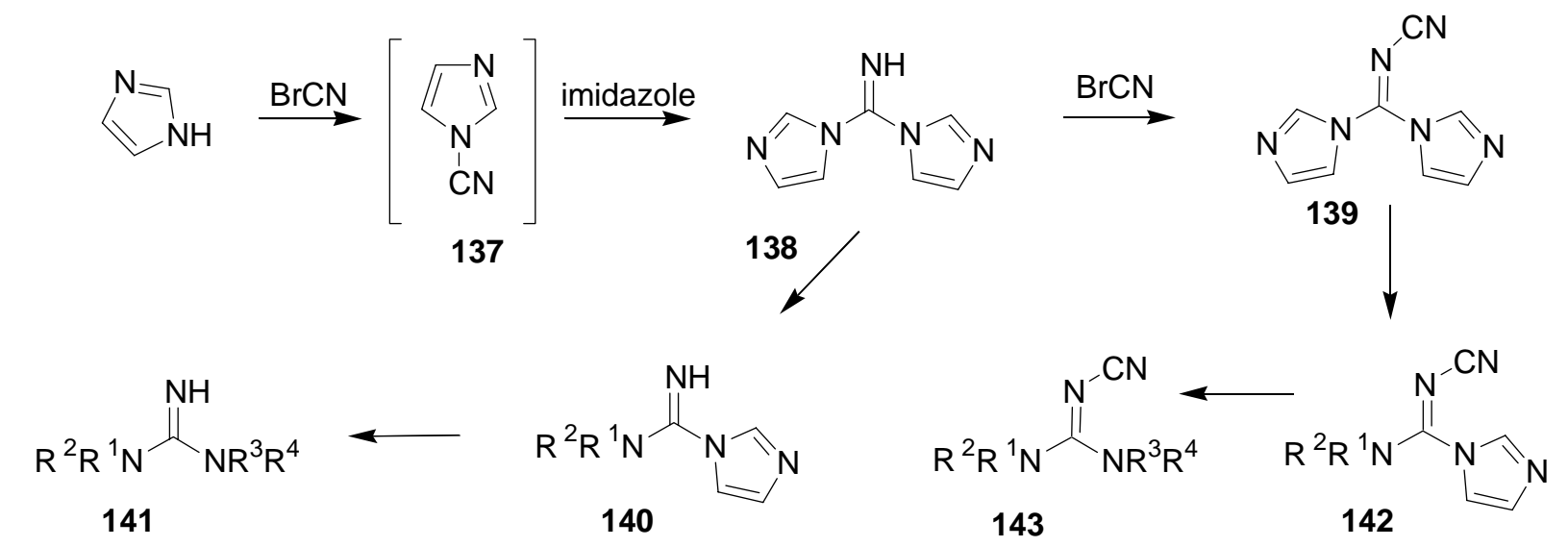

\section{Scheme 47}


Table 39. Preparation of Guanidines 141 and cyanoguanidines 143 (of Scheme 47)

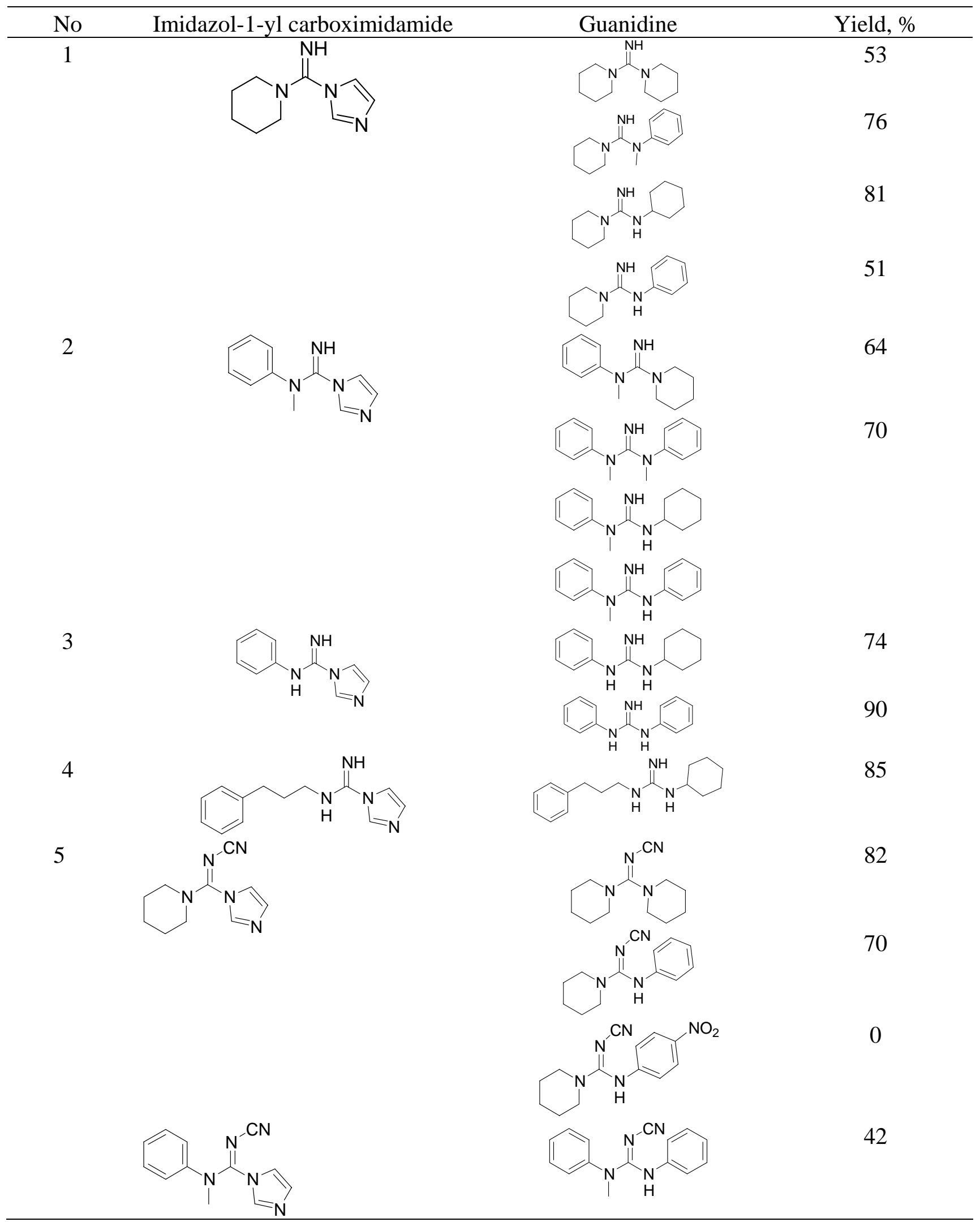


Benzotriazolylcarboximidoyl chlorides 145 (stable, odorless and convenient to handle) also allow the preparation of unsymmetrical guanidines ${ }^{57}$ (Scheme 48).
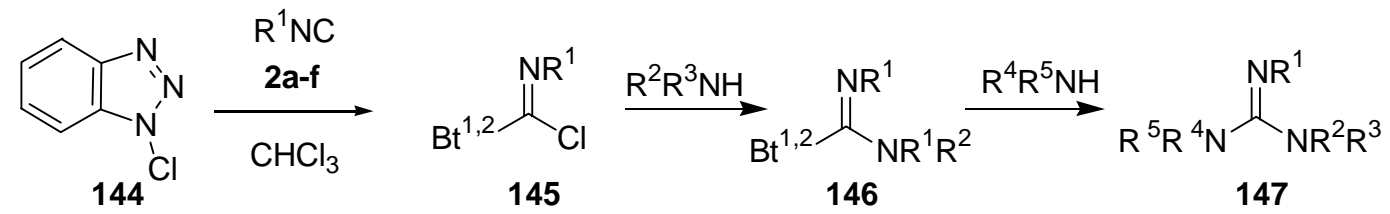

\section{Scheme 48}

\section{Conclusions}

We have attempted to summarize recent advances in guanidine synthesis from the point of view of guanylating agents.

\section{Supplementary information is available}

\section{References}

1. (a) Kovacevic, B.; Maksic, Z. B. Org. Lett. 2001, 3, 1523. (b) Hannon, C. L.; Anslyn, E. V. Bioorganic Chemistry Frontiers 1993, 3, 193.

2. Tapiero, H.; Mathe, G.; Couvreur, P.; Tew, K. D. Biomed. Pharmacother. 2002, 56, 439.

3. (a) Berlinck, R. G. S. Progr. Chem. Org. Nat. Prod. 1995, 66, 119. (b) Berlinck, R. G. S. Nat. Prod. Res. 1996, 13, 377. (c) Berlinck, R. G. S. Nat. Prod. Res. 1999, 16, 339. (d) Berlinck, R. G. S. Nat. Prod. Res. 2002, 19, 617. (e) Heys, L.; Moore, C. G.; Murphy, P. J. Chem. Soc Rev. 2000, 29, 57. (f) Hanai, T.; Inamaoto, Y.; Inamoto, S. J. Chromatogr. 2000, 747, 123.

4. (a) Albert, M.; Feiertag, P.; Hayn, G.; Saf, R.; Honig, H. Biomacromolecules 2003, 4, 1811.

(b) Goessnitzer, E.; Punkenhofer, A.; Ryder, N. S. Arc. Pharm. 2003, 336, 336.

5. De Simone, G.; Menchise, V.; Omaggio, S.; Pedone, C.; Scozzafava, A.; Supuran, C. T. Biochemistry 2003, 42, 9013.

6. (a) For recent review, see Masereel, B.; Pochet, L.; Laeckmann, D. Eur. J. Med. Chem. 2003, 38, 547. (b) Bao, X.-H.; Lu, W.-C.; Liu, L.; Chen, N.-Y. Acta Pharmacol. Sinica 2003, 24, 472.

7. (a) Kralova, J.; Dvorak, M.; Kral, V. J. Med. Chem. 2003, 46, 2049. (b) Wright, L. R.; Rothbard, J. B.; Wender, P. A. Curr. Prot. Pept. Sci. 2003, 4, 105. (c) Orner, B. P.; Hamilton, A. D. J. Incl. Phen. Macrocycl. Chem. 2001, 41, 141. (d) Masuda, T.; Shibuya, S.; Arai, M.; Yoshida, S.; Tomozawa, T.; Ohno, A.; Yamashita, M.; Honda, T. Bioorg. Med. Chem. Lett. 2003, 13, 669. 
8. (a) Tinti, J.-M.; Nofre, C. Design of Sweeteners In Sweeteners: Discovery, Molecular Design and Chemoreseption, Walters, D. E.; Orthoefer, F. T.; DuBois, G. E. Eds.; ACS: Washington, D.C. 1991; pp 88-112. (b) Glaser, D. P. Appl. Chem. 2002, 74, 1153. (c) Katritzky, A. R.; Petrukhin, R.; Perumal, S.; Karelson, M.; Prakash, I.; Desai, N. Croat. Chem. Acta 2002, 75, 475. (d) Nagarajan, S.; Kellogg, M. S.; DuBois, G. E.; Hellekant, G. J. Med. Chem. 1996, 39, 4167. (e) Droupadi, P. R.; Linthicum, D. S. Int. J. Biochem. Cell. Biol. 1995, 27, 351.

9. Costa, M.; Chiusoli, G. P.; Taffurelli, D.; Dalmonego, G. J. Chem. Soc., Perkin Trans. 1 1998, 1541.

10. (a) Ishikawa, T.; Isobe, T. Chem. Eur. J. 2002, 8, 553. (b) McManus, J. C.; Genski, T.; Carey, J. S.; Taylor, R. J. K. Synlett 2003, 369. (c) McManus, J. C.; Carey, J. S.; Taylor, R. J. K. Synlett 2003, 365.

11. (a) Manimala, J. C.; Anslyn, E. V. Eur. J. Org. Chem. 2002, 3909. (b) Solid Phase synthesis of Guanidines, Burgess, K.; Chen, J. In Solid Phase Organic Synthesis, Burgess, K. Ed.; John Wiley \& Sons, Inc.: New York, 2000, pp 1-23. (c) Schneider, S. E.; Bishop, P. A.; Salazar, M. A.; Bishop, O. A.; Anslyn, E. V. Tetrahedron 1998, 54, 15063.

12. Ramadas, K.; Srinivasan, N. Tetrahedron Lett. 1995, 36, 2841.

13. Yong, Y. F.; Kowalski, J. A.; Lipton, M. A. J. Org. Chem. 1997, 62, 1540.

14. Manimala, J. C.; Anslyn, E. V. Tetrahedron Lett. 2002, 43, 565.

15. Kim, K. S.; Qian, L. Tetrahedron Lett. 1993, 34, 7677.

16. (a) Levallet, C.; Lerpiniere, J.; Ko, S. Y. Tetrahedron 1997, 53, 5291. (b) Dahmen, S.; Bräse, S. Org. Lett. 2000, 2, 3563.

17. Linton, B. R.; Carr, A. J.; Orner, B. P.; Hamilton, A. D. J. Org. Chem. 2000, 65, 1566.

18. Jirgensons, A.; Kums, I.; Kauss, V.; Kalvins, I. Synth. Commun. 1997, 27, 315.

19. Li, M.; Wilson, L. J.; Portlock, D. E. Tetrahedron Lett. 2001, 42, 2273.

20. Guisado, O.; Martinez, S.; Pastor, J. Tetrahedron Lett. 2002, 43, 7105.

21. (a) Kilburn, J. P. Lau, J.; Jones, R. C. F. Tetrahedron 2002, 58, 1739. (b) Zhang, J.; Shi, Y.; Stein, P.; Atwal, K.; Li, C. Tetrahedron Lett. 2002, 43, 57.

22. Kent, D. R.; Cody, W. L.; Doherty, A. M. Tetrahedron Lett. 1996, 37, 8711.

23. Okajima, N.; Okada, Y. J. Het. Chem. 1991, 28, 177.

24. Rasmussen, C. R.; Villani Jr., F. J.; Reynolds, B. E.; Plampin, J. N.; Hood, A. R.; Hecker, L. R.; Nortey, S. O.; Hanslin, A.; Constanzo, M. J.; Howse Jr., R. M.; Molinari, A. J. Synthesis 1988, 460.

25. Chandrakumar, N. S. Synth. Commun. 1996, 26, 2613.

26. Lal, B.; Gangopadhyay, A. K. Tetrahedron Lett. 1996, 37, 2483

27. Yuan, C.; Williams, R. M. Tetrahedron Lett. 1996, 37, 1945.

28. (a) Lin, P.; Ganesan, A. Tetrahedron Lett. 1998, 39, 9789. (b) Dodd, D. S.; Zhao, Y. Tetrahedron Lett. 2001, 42, 1259.

29. Guo, Z.-X.; Cammidge, A. N.; Horwell, D. C. Synth. Commun. 2000, 30, 2933.

30. Lin, P.; Heng, S. H. C.; Sim, M. M. Synthesis 2003, 255.

31. Gomez, L.; Gellibert, F.; Wagner, A.; Mioskowski, C. Chem. Eur. J. 2000, 6, 4016.

32. Dodd, D. S.; Wallace, O. W. Tetrahedron Lett. 1998, 39, 5701.

33. Kearney, P. C.; Fernandez, M.; Flygare, J. A. Tetrahedron Lett. 1998, 39, 2663. 
34. Novak, L.; Hanania, M.; Kovacs, P.; Kovacs, C. E.; Kolonits, P.; Szantay, C. Synth. Commun. 1999, 29, 1757.

35. Reiter, L. A.; Brighty, K. E.; Bryant, R. A.; Goldsmith, M. E. Synth. Commun. 1996, 26, 1423.

36. Molina, P.; Aller, E.; Larenzo, A. Synlett 2003, 714.

37. Chen, J.; Pattarawarapan, M.; Zhang, A. J.; Burgess, K. J. Comb. Chem. 2000, 2, 276.

38. Drewry, D. H.; Gerritz, S. W.; Linn, J. A. Tetrahedron Lett. 1997, 38, 3377.

39. Reddy, N. L.; Fan, W.; Magar, S. S.; Perlman, M. E.; Yost, E.; Zhang, L.; Berlove, D.; Fischer, J. B.; Burke-Howie, K.; Wolcott, T.; Durant, G. J. J. Med. Chem. 1998, 41, 3298.

40. (a) Scott, F. L.; O’Donovan, D. G.; Reilly, J. J. Am. Chem. Soc. 1953, 75, 4053. (b) Brederec, H.; Effenberger, F.; Hajek, M. Chem. Ber 1965, 98, 3178. (c) Bernatowicz, M. S.; Wu, Y.; Matsueda, G. R. J. Org. Chem. 1992, 57, 2497.

41. Yong, Y. F.; Kowalski, J. A.; Thoen, J. C.; Lipton, M. A. Tetrahedron Lett. 1999, 40, 53.

42. Zhang, Y.; Kennan, A. J. Org. Lett. 2001, 3, 2341.

43. Drake, B.; Patek, M.; Lebl, M. Synthesis 1994, 579.

44. Ghosh, A. K.; Hol, W. G.; Fan, E. J. Org. Chem. 2001, 66, 2161.

45. Feichtinger, K.; Zapf, C.; Singh, H. L.; Goodman, M. J. Org. Chem. 1998, 63, 3804.

46. Feichtinger, K.; Singh, H. L.; Mattews, K.; Goodman, M. J. Org. Chem. 1998, 63, 8432

47. (a) Hui, Y.; Ptak, R.; Paulman, R.; Pallasch, M.; Chang, C.-W. T. Tetrahedron Lett. 2002, 43, 9255. (b) Sun, C.-M.; Shey, J.-Y. J. Comb. Chem. 1999, 1, 361.

48. Baker, T. J.; Goodman, M. Synthesis 1999, 1423.

49. Maryanoff, C. A.; Stanzione, R. C.; Plampin, J. N.; Mills, J. E. J. Org. Chem. 1986, 51, 1882.

50. Miller, A. E.; Bischoff, J. J. Synthesis 1996, 777.

51. Ramadas, K.; Janarthanan, N.; Pritha, R. Synlett 1997, 1053.

52. Srinivasan, N.; Ramadas, K. Tetrahedron Lett. 2001, 42, 343.

53. Katritzky, A. R.; Parris, R. L.; Allin, S. M.; Steel, P.J. Synth. Commun. 1995, 25, 1173.

54. Musiol, H.-J.; Moroder, L. Org. Lett. 2001, 3, 3859.

55. Katritzky, A. R.; Rogovoy, B. V.; Chassaing, C.; Vvedensky, V. J. Org. Chem. 2000, 65, 8080.

56. Wu, Y.-Q.; Hamilton, S. K.; Wilkinson, D. E.; Hamilton, G. S. J. Org. Chem. 2002, 67, 7553.

57. Katritzky, A. R.; Rogovoy, B.; Klein, C.; Insuasty, H.; Vvedensky, V.; Insuasty, B. J. Org. Chem. 2001, 66, 2854. 


\section{Biographical sketch}

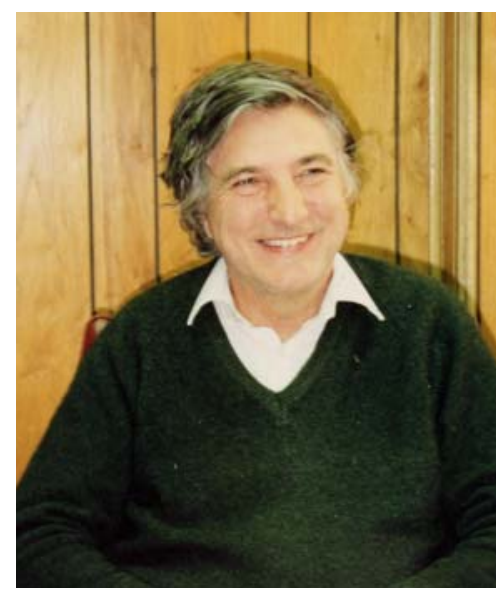

Alan Katritzky has taught, researched, consulted and published on heterocyclic chemistry, synthetic methods, and QSPR, first in the UK and since 1980 at the University of Florida where he is Kenan Professor of Chemistry and Director of the Center for Heterocyclic Compounds.

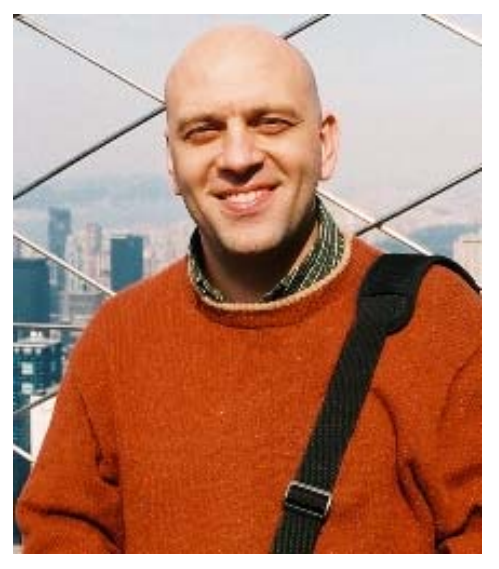

Dr. Rogovoy was born January $18^{\text {th }}$, 1973. He received his MS degree in 1995 in Chemical Engineering at Ukrainian State University for Chemical Engineering, Dnepropetrovsk, Ukraine and PhD degree in 2002 at the University of Tartu, Tartu, Estonia. In 1998 Dr. Rogovoy joined the Center for Heterocyclic Compounds at the University of Florida. In 2003 he joined the Department of Pharmaceutical Chemistry in School of Pharmacy in Rutgers University where he was involved in a development of prodrug systems for the site-specific release and activation of anticancer drugs and improvement of therapeutic effectiveness of anticancer agents with reduced systemic toxicity. In November of 2004, Dr. Rogovoy obtained a position of Senior Research Scientist at ChemDiv Inc, San Diego, CA. His research interests include heterocyclic synthesis, combinatorial synthesis in solution and solid support, development of efficient synthetic approaches. 